\title{
A Neumann series of Bessel functions representation for solutions of perturbed Bessel equations
}

\author{
Vladislav V. Kravchenko ${ }^{1}$, Sergii M. Torba ${ }^{1}$ and Raúl Castillo-Pérez ${ }^{2}$ \\ ${ }^{1}$ Departamento de Matemáticas, CINVESTAV del IPN, Unidad Querétaro, \\ Libramiento Norponiente \#2000, Fracc. Real de Juriquilla, Querétaro, Qro., 76230 MEXICO. \\ 2 Maestría en Telecomunicaciones, ESIME Zacatenco, Instituto Politécnico Nacional, \\ Av. Instituto Politécnico Nacional S/N, D.F. Mexico, 07738 MEXICO. \\ e-mail: vkravchenko@math.cinvestav.edu.mx, storba@math.cinvestav.edu.mx,rcastillo@ipn.mx*
}

October 6, 2018

\begin{abstract}
A new representation for a regular solution of the perturbed Bessel equation of the form $L u=-u^{\prime \prime}+\left(\frac{l(l+1)}{x^{2}}+q(x)\right) u=\omega^{2} u$ is obtained. The solution is represented as a Neumann series of Bessel functions uniformly convergent with respect to $\omega$. For the coefficients of the series explicit direct formulas are obtained in terms of the systems of recursive integrals arising in the spectral parameter power series (SPPS) method, as well as convenient for numerical computation recurrent integration formulas.

The result is based on application of several ideas from the classical transmutation (transformation) operator theory, recently discovered mapping properties of the transmutation operators involved and a Fourier-Legendre series expansion of the transmutation kernel. For convergence rate estimates, asymptotic formulas, a Paley-Wiener theorem and some results from constructive approximation theory were used.

We show that the analytical representation obtained among other possible applications offers a simple and efficient numerical method able to compute large sets of eigendata with a nondeteriorating accuracy.
\end{abstract}

\section{Introduction}

In the present work the equation

$$
-u^{\prime \prime}+\left(\frac{l(l+1)}{x^{2}}+q(x)\right) u=\omega^{2} u, \quad x \in(0, b]
$$

is studied, where $l$ is a real number, $l \geq-\frac{1}{2}, q$ is a complex-valued function on $[0, b]$ satisfying the following condition

$$
\begin{aligned}
& x q(x) \in L_{1}(0, b) \quad \text { if } l>-1 / 2, \\
& x^{1-\varepsilon} q(x) \in L_{1}(0, b) \quad \text { for some } \varepsilon>0 \text { if } l=-1 / 2 \text {, }
\end{aligned}
$$

${ }^{*}$ Research was supported by CONACYT, Mexico via the projects 166141 and 222478. R. Castillo would like to thank the support of CONACYT and of the SIBE and EDI programs of the IPN as well as that of the project SIP 20160525. 
and $\omega$ is a (complex) spectral parameter. Denote $L=-\frac{d^{2}}{d x^{2}}+\frac{l(l+1)}{x^{2}}+q(x)$. Equations of the form (1.1) appear naturally in many real-world applications after a separation of variables and therefore have received considerable attention (see, e.g., [5], 6], 77, 8], 12, [16, [25, Sect. 3.7], 35]).

The main result of the work is a representation of a regular solution $u_{l}(\omega, x)$ of (1.1) satisfying the asymptotic relation $u_{l}(\omega, x) \sim x^{l+1}$ when $x \rightarrow 0$ in the form of the following series of Bessel functions

$$
u_{l}(\omega, x)=\frac{2^{l+1} \Gamma\left(l+\frac{3}{2}\right)}{\sqrt{\pi} \omega^{l}} x j_{l}(\omega x)+\sum_{n=0}^{\infty}(-1)^{n} \beta_{n}(x) j_{2 n}(\omega x)
$$

where $j_{k}$ denotes the spherical Bessel function of the first kind of order $k$. For the coefficients $\beta_{n}$ explicit direct formulas are obtained in terms of a system of recursive integrals arising in the SPPS method [6]. For a fixed $x$ the series in (1.3) represents a so-called Neumann series of Bessel functions (see [34, 36] and a recent publication on the subject [3] and references therein).

We prove that the series (1.3) converges uniformly with respect to $\omega$. More precisely, the very convenient estimates (5.3) and (5.4) are obtained which guarantee that a partial sum from (1.3) approximates equally well the solution $u_{l}(\omega, x)$ both for small and for large values of the spectral parameter $\omega$. We illustrate this feature of (1.3) with several numerical examples which show that this new representation besides other possible applications can be used as a simple and powerful numerical method for solving boundary value and spectral problems related to (1.1).

In the recent work [18 an analogous representation of solutions was obtained for the regular one-dimensional Schrödinger equation $-y^{\prime \prime}+q(x) y=\omega^{2} y$. However following similar lines does not lead to the result in the case of the perturbed Bessel equation. Several new ideas are necessary. We make use of the properties of a couple of transmutation operators, one of them relating the operators $\frac{d^{2}}{d x^{2}}$ and $\frac{d^{2}}{d x^{2}}-\frac{l(l+1)}{x^{2}}$ (studied in [29], [28], [20]) and the other relating the operators $\frac{d^{2}}{d x^{2}}-\frac{l(l+1)}{x^{2}}$ and $\frac{d^{2}}{d x^{2}}-\frac{l(l+1)}{x^{2}}-q(x)$ (studied in [33], [9]). The first of these transmutations is used for separating the part corresponding to the unperturbed equation $(q \equiv 0)$ and the second to add the perturbation. We specify that neither here nor in the title of the paper the perturbation means any kind of smallness of the coefficient $q$. The use of two transmutation operators allows us to show that the regular solution of (1.1) can be represented in the form

$$
u_{l}(\omega, x)=\frac{2^{l+1} \Gamma\left(l+\frac{3}{2}\right)}{\sqrt{\pi} \omega^{l}} x j_{l}(\omega x)+\int_{0}^{x} R(x, t) \cos \omega t d t
$$

where the kernel $R(x, t)$ is a sufficiently good function which admits a convergent Fourier-Legendre series expansion. Moreover, this approach makes it possible to write down the result of the integral $\int_{0}^{x} R(x, t) t^{2 k} d t$ for any $k=0,1,2, \ldots$ and as a consequence to obtain explicit formulas for the coefficients of the Fourier-Legendre series. Substitution of the series into (1.4) leads to the main result (1.3). The convergence rate of the Fourier-Legendre series (and, consequently, of the series (1.3) ) depends on the smoothness of the integral kernel $R$. Only few basic properties of the kernel $R$ can be obtained using the results from [33] and [9]. We implement a different approach based on the asymptotic formulas from [15] and [11], a Paley-Wiener theorem and the constructive approximation theory [10]. As a result, we present close to optimal convergence rate estimates depending on the parameter $l$ and the smoothness of the potential $q$.

Analogous formulas are developed for the derivative of the regular solution.

The direct explicit formula for the coefficient $\beta_{n}$ is not however the most convenient for numerical computation. This is due to the fact that it involves coefficients of the Legendre polynomial of order $2 n$ which grow rapidly when $n$ grows and hence, although the coefficients $\beta_{n}$ decrease, one needs to compute linear combinations of large numbers. This reduces considerably the number of the coefficients which can be computed in machine precision. Fortunately, there exists another way to 
compute the coefficients $\beta_{n}$ with the aid of a recurrent integration procedure similar to that arising in the SPPS method [19, [17] and much more stable in practice. To develop the procedure we find the sequence of differential equations satisfied by the coefficients $\beta_{n}$. This is done by substitution of (1.3) into (1.1).

In the last part of the paper we show that (1.3) offers a simple and powerful numerical method for computing regular solutions of (1.1) and for solving spectral problems related. The uniform convergence with respect to $\omega$ allows one to compute large sets of eigendata with a non-deteriorating accuracy.

\section{From regular to singular}

Consider the following integral operator defined on $C[0, b]$,

$$
Y_{l} v(x):=\frac{x^{-l}}{2^{l+\frac{1}{2}} \Gamma\left(l+\frac{3}{2}\right)} \int_{0}^{x}\left(x^{2}-s^{2}\right)^{l} v(s) d s .
$$

The following statement is a slightly precised result from [29].

Proposition 2.1 ([28, 20]). Let $v \in C^{2}[0, b]$ and $v^{\prime}(0)=0$. Then

$$
\left(\frac{d^{2}}{d x^{2}}-\frac{l(l+1)}{x^{2}}\right) Y_{l} v=Y_{l} \frac{d^{2}}{d x^{2}} v
$$

In particular [20],

$$
Y_{l}: x^{2 k} \mapsto \frac{\Gamma\left(k+\frac{1}{2}\right) \Gamma(l+1)}{2^{l+\frac{3}{2}} \Gamma\left(l+\frac{3}{2}\right) \Gamma\left(k+l+\frac{3}{2}\right)} x^{2 k+l+1}, \quad k=0,1,2, \ldots
$$

Denote

$$
b_{l}(\omega x):=\sqrt{\omega x} J_{l+\frac{1}{2}}(\omega x) .
$$

This function is a regular solution of the equation

$$
\left(\frac{d^{2}}{d x^{2}}-\frac{l(l+1)}{x^{2}}\right) v=-\omega^{2} v, \quad x \in(0, b] .
$$

Its corresponding power series has the form

$$
b_{l}(\omega x)=(\omega x)^{l+1} \sum_{k=0}^{\infty} \frac{(-1)^{k}(\omega x)^{2 k}}{2^{2 k+l+\frac{1}{2}} \Gamma(k+1) \Gamma\left(k+l+\frac{3}{2}\right)} .
$$

Remark 2.2. From (2.1) we obtain

$$
Y_{l}[\cos \omega x]=\frac{\sqrt{\pi} \Gamma(l+1)}{2 \omega^{l+1} \Gamma\left(l+\frac{3}{2}\right)} b_{l}(\omega x) .
$$




\section{Transmutation of Bessel-type operators}

Throughout this section we assume that $q \in C[0, b]$. In [33] the existence of a unique continuous kernel $V(x, t)$ was proved such that for all $\omega \in \mathbb{C}$ the function

$$
u(\omega, x)=\mathcal{T}\left[b_{l}(\omega x)\right]:=b_{l}(\omega x)+\int_{0}^{x} V(x, t) b_{l}(\omega t) d t
$$

is a regular solution of the equation

$$
\left(\frac{d^{2}}{d x^{2}}-\frac{l(l+1)}{x^{2}}-q(x)\right) u=-\omega^{2} u, \quad x \in(0, b]
$$

and

$$
V(x, x)=\frac{Q(x)}{2}
$$

where $Q(x):=\int_{0}^{x} q(t) d t$, see also 9$]$.

If $l \neq 0$ then the left endpoint is singular. Despite that, the equation $L u=0$ possesses a solution $\phi(x)$ which is bounded at $x=0$ and satisfies the following asymptotics at $x=0$

$$
\begin{aligned}
\phi(x) & \sim x^{l+1}, \quad x \rightarrow 0, \\
\phi^{\prime}(x) & \sim(l+1) x^{l}, \quad x \rightarrow 0,
\end{aligned}
$$

see, e.g., [16, Lemma 3.2] for a real-valued q. In [6] an explicit construction of the solution with this asymptotics at zero for the general case of a complex-valued $q$ was given.

From now on we assume that there exists a non-vanishing on $(0, b]$ complex-valued solution $u_{0}$ of the equation

$$
-u_{0}^{\prime \prime}+\left(\frac{l(l+1)}{x^{2}}+q(x)\right) u_{0}=0
$$

satisfying together with its first derivative the asymptotic relations (3.3) and (3.4). In [6] the existence and a procedure for construction of such a solution was given in the case when $q(x) \geq 0$, $x \in(0, b]$.

Notation 3.1. Let us define the following system of functions

$$
\varphi_{n}(x):=(-1)^{n}(2 n) ! u_{0}(x) \widetilde{X}^{(2 n)}(x)
$$

where

$$
\begin{aligned}
\tilde{X}^{(0)} & \equiv 1, \\
\widetilde{X}^{(n)}(x) & = \begin{cases}\int_{0}^{x} u_{0}^{2}(t) \widetilde{X}^{(n-1)}(t) d t, & \text { if } n \text { is odd, } \\
-\int_{0}^{x} \frac{\widetilde{X}^{(n-1)}(t)}{u_{0}^{2}(t)} d t, & \text { if } n \text { is even. }\end{cases}
\end{aligned}
$$

We keep the notation $\widetilde{X}$ for consistency with other publications on the SPPS method, see, e.g., [19], [17], 21].

In [6] it was proved that

$$
\mathcal{T}\left[x^{2 k+l+1}\right]=(-1)^{k} 2^{2 k} k !\left(l+\frac{3}{2}\right)_{k} u_{0}(x) \widetilde{X}^{(2 k)}(x) \quad \text { for any } k=0,1,2, \ldots
$$


Theorem 3.2. Let $q \in C[0, b], l \geq-\frac{1}{2}$. There exists a continuous function $R^{(0)}(x, t), 0 \leq t \leq x \leq b$ such that for any $\omega \in \mathbb{C}$ the function

$$
u(\omega, x)=a(\omega) b_{l}(\omega x)+\int_{0}^{x} R^{(0)}(x, t) \cos \omega t d t
$$

with

$$
a(\omega)=\frac{\sqrt{\pi} \Gamma(l+1)}{2 \omega^{l+1} \Gamma\left(l+\frac{3}{2}\right)}
$$

is a regular solution of (3.1).

Proof. Consider

$$
u(\omega, x)=\mathcal{T} Y_{l}[\cos \omega x]=a(\omega) b_{l}(\omega x)+\int_{0}^{x} V(x, t) Y_{l}[\cos \omega t] d t .
$$

This function is a solution of (3.1) due to the fact that $Y_{l}[\cos \omega t]=a(\omega) b_{l}(\omega x)$. Hence it is sufficient to prove that (3.9) can be written in the form (3.8).

Consider

$$
\begin{aligned}
\int_{0}^{x} V(x, t) Y_{l}[\cos \omega t] d t & =\frac{1}{2^{l+\frac{1}{2}} \Gamma\left(l+\frac{3}{2}\right)} \int_{0}^{x} V(x, t) t^{-l} \int_{0}^{t}\left(t^{2}-s^{2}\right)^{l} \cos \omega s d s d t \\
& =\frac{1}{2^{l+\frac{1}{2}} \Gamma\left(l+\frac{3}{2}\right)} \int_{0}^{x} \cos \omega s \int_{s}^{x} V(x, t) t^{-l}\left(t^{2}-s^{2}\right)^{l} d t d s
\end{aligned}
$$

Denote

$$
R^{(0)}(x, s)=\frac{1}{2^{l+\frac{1}{2}} \Gamma\left(l+\frac{3}{2}\right)} \int_{s}^{x} V(x, t) t^{-l}\left(t^{2}-s^{2}\right)^{l} d t=\frac{1}{2^{l+\frac{1}{2}} \Gamma\left(l+\frac{3}{2}\right)} \int_{s}^{x} V(x, t)\left(t-\frac{s^{2}}{t}\right)^{l} d t .
$$

Since $V$ is continuous, $R^{(0)}$ is continuous as well.

The solution (3.8) can be written in the form of an SPPS [6],

$$
u(\omega, x)=\frac{\sqrt{\pi} \Gamma(l+1)}{2^{l+\frac{3}{2}} \Gamma^{2}\left(l+\frac{3}{2}\right)} u_{0}(x) \sum_{k=0}^{\infty} \omega^{2 k} \widetilde{X}^{(2 k)}(x) .
$$

Indeed, we have that

$$
\begin{aligned}
u(\omega, x) & =\frac{a(\omega)}{2^{l+\frac{1}{2}}} \sum_{k=0}^{\infty} \frac{(-1)^{k} \omega^{2 k+l+1}}{2^{2 k} \Gamma(k+1) \Gamma\left(k+l+\frac{3}{2}\right)} \mathcal{T}\left[x^{2 k+l+1}\right] \\
& =\frac{a(\omega)}{2^{l+\frac{1}{2}}} \sum_{k=0}^{\infty} \frac{\omega^{2 k+l+1}\left(l+\frac{3}{2}\right)_{k} u_{0}(x) \widetilde{X}^{(2 k)}(x)}{\Gamma\left(k+l+\frac{3}{2}\right)}
\end{aligned}
$$

where we used (3.7). Taking into account the definition of $a(\omega)$ and the identity $\left(l+\frac{3}{2}\right)_{k}=$ $\Gamma\left(k+l+\frac{3}{2}\right) / \Gamma\left(l+\frac{3}{2}\right)$ we obtain (3.11). Thus,

$$
\begin{aligned}
u_{0}(x) \sum_{k=0}^{\infty} \omega^{2 k} \widetilde{X}^{(2 k)}(x) & =\frac{2^{l+\frac{3}{2}} \Gamma^{2}\left(l+\frac{3}{2}\right)}{\sqrt{\pi} \Gamma(l+1)} a(\omega) b_{l}(\omega x)+\int_{0}^{x} R(x, t) \cos \omega t d t \\
& =\frac{2^{l+\frac{1}{2}} \Gamma\left(l+\frac{3}{2}\right)}{\omega^{l+1}} b_{l}(\omega x)+\int_{0}^{x} R(x, t) \sum_{k=0}^{\infty} \frac{(-1)^{k}(\omega t)^{2 k}}{(2 k) !} d t
\end{aligned}
$$


where

$$
R(x, t):=\frac{2^{l+\frac{3}{2}} \Gamma^{2}\left(l+\frac{3}{2}\right)}{\sqrt{\pi} \Gamma(l+1)} R^{(0)}(x, t) .
$$

Hence,

$$
u_{0}(x) \sum_{k=0}^{\infty} \omega^{2 k} \widetilde{X}^{(2 k)}(x)=\Gamma\left(l+\frac{3}{2}\right) \sum_{k=0}^{\infty} \frac{(-1)^{k} \omega^{2 k} x^{2 k+l+1}}{2^{2 k} \Gamma(k+1) \Gamma\left(k+l+\frac{3}{2}\right)}+\sum_{k=0}^{\infty} \frac{(-1)^{k} \omega^{2 k}}{(2 k) !} \int_{0}^{x} R(x, t) t^{2 k} d t .
$$

The coefficients of the equal uniformly convergent power series with respect to $\omega$ must coincide, thus,

$$
u_{0}(x) \widetilde{X}^{(2 k)}(x)=\frac{(-1)^{k} \Gamma\left(l+\frac{3}{2}\right) x^{2 k+l+1}}{2^{2 k} \Gamma(k+1) \Gamma\left(k+l+\frac{3}{2}\right)}+\frac{(-1)^{k}}{(2 k) !} \int_{0}^{x} R(x, t) t^{2 k} d t .
$$

Hence

$$
\varphi_{k}(x)=c_{k, l} x^{2 k+l+1}+\int_{0}^{x} R(x, t) t^{2 k} d t \quad \text { for any } k=0,1,2, \ldots
$$

where

$$
c_{k, l}:=\frac{\Gamma\left(l+\frac{3}{2}\right) \Gamma\left(k+\frac{1}{2}\right)}{\sqrt{\pi} \Gamma\left(k+l+\frac{3}{2}\right)},
$$

and we used the identities $(2 k) !=\Gamma(2 k+1)=k 2^{2 k} \Gamma(k) \Gamma\left(k+\frac{1}{2}\right) / \sqrt{\pi}$.

\section{A Fourier-Legendre representation of the kernel $R(x, t)$}

Multiplication of equality (3.8) by the constant $\frac{2^{l+\frac{3}{2}} \Gamma^{2}\left(l+\frac{3}{2}\right)}{\sqrt{\pi} \Gamma(l+1)}$ from $(\underline{3.12})$ allows us to write down a regular solution of (3.1) in the form

$$
u_{l}(\omega, x)=d(\omega) b_{l}(\omega x)+\int_{0}^{x} R(x, t) \cos \omega t d t
$$

with $d(\omega):=\frac{2^{l+\frac{1}{2}} \Gamma\left(l+\frac{3}{2}\right)}{\omega^{l+1}}$. It is related with the solution from Theorem 3.2 by $u_{l}(\omega, x)=\frac{2^{l+\frac{3}{2}} \Gamma^{2}\left(l+\frac{3}{2}\right)}{\sqrt{\pi} \Gamma(l+1)} u(\omega, x)$ and for any $\omega$ satisfies the following asymptotic equality when $x \rightarrow 0, u_{l}(\omega, x) \sim x^{l+1}$. As a next step we construct a Fourier-Legendre representation for the kernel $R(x, t)$.

First, we need to recall the following notations, c.f. [10, Chap. 2, $\S 7$ and $\S 9$ ]. For $\alpha>0$ we write $\alpha=r+\beta$, where $r \in \mathbb{Z}$ and $0<\beta \leq 1$, and say that a function $f$ belongs to $\operatorname{Lip}_{\alpha}(I)$ class, with $I$ being either a segment or the whole line, if $f \in C^{r}(I)$ and $f^{(r)} \in \operatorname{Lip}_{\beta}(I)$. Consider the difference operator $\Delta_{h}: L_{p}(I) \rightarrow L_{p}\left(I_{h}\right)$ acting on a function $f$ as $\Delta_{h} f(\cdot)=f(\cdot+h)-f(\cdot)$, here $I_{h}:=[a, b-h]$ if $I=[a, b], h<b-a$ and $I_{h}:=I$ if $I=\mathbb{R}$. Then the $r$-th modulus of smoothness of $f$ is defined by

$$
\omega_{r}(f, t)_{L_{p}(I)}:=\sup _{0<h \leq t}\left\|\Delta_{h}^{r}(f)\right\|_{L_{p}\left(I_{r h}\right)} .
$$

For $\alpha>0$ let $r$ be the smallest integer satisfying $r>\alpha$, i.e., $r=[\alpha]+1$. Then the generalized Lipschitz class $\operatorname{Lip}_{\alpha}^{*}(I, p)$ is defined as the class of functions $f \in L_{p}(I)$ satisfying $\omega_{r}(f, t)_{L_{p}(I)} \leq M t^{\alpha}$ for all $t>0$ with some constant $M=M(f)$. By $W_{2}^{\alpha}(\mathbb{R}), \alpha \geq 0$ we denote the fractional-order Sobolev space, also called Bessel potential space [2, Chap. 7] consisting of the functions satisfying $f \in L_{2}(\mathbb{R})$ and $\left(1+|\xi|^{2}\right)^{\alpha / 2} \mathcal{F}[f](\xi) \in L_{2}(\mathbb{R})$, where $\mathcal{F}$ is the Fourier transform operator.

Following [15] we introduce the notation

$$
\tilde{q}(x)= \begin{cases}|q(x)|, & l>-1 / 2 \\ (1-\log (x / b))|q(x)|, & l=-1 / 2\end{cases}
$$


Proposition 4.1. Let $q$ satisfy the condition (1.2). Suppose additionally that

$$
x^{\alpha} \tilde{q}(x) \in L_{1}(0, b) \quad \text { for some } \alpha \in[0,1], \alpha<3 / 2+l .
$$

Let $x>0$ be fixed. Then there exists an even, compactly supported on $[-x, x]$ function $\widetilde{R}(x, t)$ such that

1. $\widetilde{R} \in W_{2}^{l+3 / 2-\alpha-\varepsilon}(\mathbb{R})$ for any sufficiently small $\varepsilon>0$; if $\alpha<l+1$ then additionally $\widetilde{R} \in$ $\operatorname{Lip}_{l+1-\alpha-\varepsilon}(\mathbb{R})$.

2. $\widetilde{R} \in \operatorname{Lip}_{l+3 / 2-\alpha}^{*}(\mathbb{R}, 2)$;

3. the function $R$ from (4.1) satisfies

$$
R(x, t)=2 \widetilde{R}(x, t), \quad 0 \leq t \leq x .
$$

Proof. Consider the function

$$
g(\omega):=u_{l}(\omega, x)-d(\omega) b_{l}(\omega x) .
$$

In [15. Lemma 2.18] it was proved under the condition $\int_{0}^{b} y \tilde{q}(y) d y<\infty$ (satisfied automatically whenever (1.2) holds) that $g(\omega)$ is an entire function and for all $\omega \in \mathbb{C}$ satisfies the following estimate

$$
|g(\omega)| \leq C\left(\frac{x}{b+|\omega| x}\right)^{l+1} e^{|\operatorname{Im} \omega| x} \int_{0}^{x} \frac{y \tilde{q}(y)}{b+|\omega| y} d y
$$

where $C=C_{l}^{2} \exp \left(C_{l} \int_{0}^{b} y \tilde{q}(y) d y\right)$ and the constant $C_{l}$ does not depend on $q$ and $x$.

Since $\frac{t}{b+|\omega| t} \leq \frac{1}{|\omega|}$, it follows from (4.2) and (4.3) that

$$
|g(\omega)| \leq \frac{C}{b^{\alpha}|\omega|^{l+2-\alpha}} \int_{0}^{x} y^{\alpha} \tilde{q}(y) d y \leq \frac{\tilde{C}}{|\omega|^{l+2-\alpha}}, \quad \omega \in \mathbb{R},
$$

showing that $g \in L^{2}(\mathbb{R})$. Applying the Paley-Wiener theorem [14, Thm. VI.7.4] we obtain that the Fourier transform of the function $g$ (which we denote by $\widetilde{R}$ ) is compactly supported on $[-x, x]$, i.e.,

$$
g(\omega)=\int_{-x}^{x} \widetilde{R}(x, t) e^{i \omega t} d t
$$

Note additionally that both functions $u_{l}(\omega, x)$ and $d(\omega) b_{l}(\omega x)$ are even functions of the real variable $\omega$, hence $\widetilde{R}$ is also even and

$$
u_{l}(\omega, x)-d(\omega) b_{l}(\omega x)=g(\omega)=2 \int_{0}^{x} \widetilde{R}(x, t) \cos \omega t d t .
$$

Since the equalities (4.1) and (4.6) hold for all $\omega$, we conclude that $2 \widetilde{R}=R$ a.e. for $0 \leq t \leq x$.

The inclusion $\widetilde{R} \in W_{2}^{l+3 / 2-\alpha-\varepsilon}(\mathbb{R})$ follows from (4.4) and from the definition of Bessel potential spaces via the Fourier transform. The inclusion $\widetilde{R} \in \operatorname{Lip}_{l+1-\alpha-\varepsilon}(\mathbb{R})$ follows from the embedding theorem $W_{2}^{\beta+1 / 2}(\mathbb{R}) \subset \operatorname{Lip}_{\beta}(\mathbb{R})$ valid for any $\beta>0, \beta \notin \mathbb{N}$, see, e.g., [32, Sect. 2.8.1].

For the last statement of the proposition we use the following generalization of [31, Theorem 85]. Let $f$ belong to $L^{2}(\mathbb{R})$ and its Fourier transform $F$ satisfies $\left(\int_{-\infty}^{-X}+\int_{X}^{\infty}\right)|F(x)|^{2} d x \leq C^{2} X^{-2 \beta}$ for some $\beta>0$ and all $X>0$. Then

$$
\omega_{r}(f, h)_{L_{2}(\mathbb{R})} \leq C_{r} h^{\beta}, \quad r=[\beta]+1 \quad \text { and } \quad C_{r}=C \sqrt{2+\frac{r}{r-\beta}} .
$$


We omit the proof of this fact since it is similar to that of [31] with the only difference that the equality $\int_{-\infty}^{\infty}\left|\Delta_{h}^{r} f(x)\right|^{2} d x=\int_{-\infty}^{\infty} \sin ^{2 r} x h \cdot|F(x)|^{2} d x$ is used. Inequality (4.4) implies that $\left(\int_{-\infty}^{-X}+\int_{X}^{\infty}\right)|g(\omega)|^{2} d \omega \leq \frac{2 \tilde{C}^{2}}{2 l+3-2 \alpha} X^{-(2 l+3-2 \alpha)}$ proving the inclusion $\widetilde{R} \in \operatorname{Lip}_{l+3 / 2-\alpha}^{*}(\mathbb{R}, 2)$.

Remark 4.2. It is possible to obtain the smoothness properties of the integral kernel $R$ directly from (3.10) and (3.12). In particular, one may verify by somewhat lengthy calculations that $\widetilde{R}$, the continuation of $R$ onto $\mathbb{R}$ as an even compactly supported function of $t$, belongs to $\operatorname{Lip}_{1+l}(\mathbb{R})$, a slight improvement as compared to Proposition 4.1. Note that compared to (1.2) the condition (4.2) does not imply additional restrictions on $q$, it only specifies the order of the singularity at zero (if any).

Let $P_{n}$ denote the Legendre polynomial of order $n, l_{k, n}$ be the corresponding coefficient of $x^{k}$, that is $P_{n}(x)=\sum_{k=0}^{n} l_{k, n} x^{k}$.

Theorem 4.3. Let $q$ satisfy (1.2). Then the kernel $R(x, t)$ has the form

$$
R(x, t)=\sum_{n=0}^{\infty} \frac{\beta_{n}(x)}{x} P_{2 n}\left(\frac{t}{x}\right)
$$

with $\beta_{n}$ being defined by the equality

$$
\beta_{n}(x)=(4 n+1) \sum_{k=0}^{n} \frac{l_{2 k, 2 n}}{x^{2 k}}\left(\varphi_{k}(x)-c_{k, l} x^{2 k+l+1}\right),
$$

and $c_{k, l}$ being given by (3.14). For any $l \geq-1 / 2$, the series in (4.8) converges in the $L_{2}$ norm.

Let additionally $q$ satisfy (4.2). If $l>\alpha-1 / 2$ then for any $x \in(0, b]$ the series in (4.8) converges uniformly with respect to $t \in[0, x]$; if $\alpha-1<l \leq \alpha-1 / 2, l \geq-1 / 2$, then for any $x \in(0, b]$ the series converges uniformly with respect to $t \in\left[0, x^{\prime}\right] \subset[0, x)$.

Let

$$
R_{N}(x, t):=\sum_{n=0}^{N} \frac{\beta_{n}(x)}{x} P_{2 n}\left(\frac{t}{x}\right) .
$$

There exist constants $C_{1}$ and $C_{2}$, dependent on $q$ and $l$ and independent of $x$ and $N$, such that for any $x>0$

$$
\left\|R(x, \cdot)-R_{N}(x, \cdot)\right\|_{L_{2}[0, x]} \leq \frac{C_{1} x^{l+3 / 2-\alpha}}{N^{l+3 / 2-\alpha}}, \quad 2 N \geq[l+5 / 2]
$$

and

$$
\left|\beta_{N}(x)\right| \leq \frac{C_{2} x^{l+2-\alpha}}{(N-1)^{l+1-\alpha}}, \quad 2 N \geq[l+9 / 2] .
$$

Proof. For any $x \in(0, b]$ the kernel $R(x, \cdot) \in L_{2}[0, x]$. Hence it admits a Fourier-Legendre series representation of the form $\sum_{j=0}^{\infty} A_{j}(x) P_{2 j}\left(\frac{t}{x}\right)$. For convenience we consider $A_{j}(x)=\frac{\beta_{j}(x)}{x}$. Note that

$$
\int_{0}^{x} R(x, t) P_{2 n}\left(\frac{t}{x}\right) d t=\sum_{j=0}^{\infty} \frac{\beta_{j}(x)}{x} \int_{0}^{x} P_{2 j}\left(\frac{t}{x}\right) P_{2 n}\left(\frac{t}{x}\right) d t=\frac{\beta_{n}(x)}{4 n+1} .
$$

Hence $\beta_{n}(x)=(4 n+1) \int_{0}^{x} R(x, t) P_{2 n}\left(\frac{t}{x}\right) d t$. On the other hand we have

$$
\int_{0}^{x} R(x, t) P_{2 n}\left(\frac{t}{x}\right) d t=\sum_{k=0}^{n} \frac{l_{2 k, 2 n}}{x^{2 k}} \int_{0}^{x} R(x, t) t^{2 k} d t=\sum_{k=0}^{n} \frac{l_{2 k, 2 n}}{x^{2 k}}\left(\varphi_{k}(x)-c_{k, l} x^{2 k+l+1}\right)
$$


where (3.13) was used. Thus, (4.9) is obtained. Note that $q$ does not need to be continuous on $[0, b]$ for the equality (3.13) to hold, the condition (1.2) is sufficient. Indeed, the functions $\varphi_{k}$ are defined by the same formulas (3.6) (their validity under the condition (1.2) can be verified similarly to [4), the SPPS representation (3.11) and the integral representation (4.1) hold, and the proof from Section 3 can be easily repeated.

Now let additionally $q$ satisfy (4.2). Consider the restriction of the function $\widetilde{R}$ from Proposition 4.1 to the segment $[-x, x]$. Since it is an even function, its Fourier-Legendre series contains only even terms and due to the equality $R(x, t)=2 \widetilde{R}(x, t), 0 \leq t \leq x$ one has $\widetilde{R}(x, t)=\sum_{n=0}^{\infty} \frac{\beta_{n}(x)}{2 x} P_{2 n}\left(\frac{t}{x}\right)$, where the series converges in $L_{2}[-x, x]$.

Theorem 4.10 from [30] states that if a function $g \in \operatorname{Lip}_{\beta}[-1,1]$, where $\beta>1 / 2$, then the partial sums of the Fourier-Legendre series of $g$ converge uniformly on $[-1,1]$ to the function $g$. By Proposition 4.1, $\widetilde{R} \in \operatorname{Lip}_{1+l-\alpha-\varepsilon}(\mathbb{R})$, hence its restriction onto $[-x, x]$ belongs to $\operatorname{Lip}_{1+l-\alpha-\varepsilon}[-x, x]$, which is sufficient to establish the uniform convergence of the series (4.8) for any $l>\alpha-1 / 2$. For $l>\alpha-1, l \geq-1 / 2$, [13, Corollary to Theorem XIII] asserts the uniform convergence of the Fourier-Legendre series of the function $\widetilde{R}$ on any $[-x+\varepsilon, x-\varepsilon] \subset(-x, x)$, i.e., the series (4.8) converges uniformly with respect to $t \in[0, x-\varepsilon] \subset[0, x)$ for any $\varepsilon>0$.

Consider the functions $g(z):=2 \widetilde{R}(x, x z)$ and $g_{N}(z):=R_{N}(x, x z), z \in[-1,1]$. The function $g_{N}$ is a polynomial of degree $2 N$ and is a partial sum of the Fourier-Legendre series of $g$, i.e., $g_{N}$ coincides with the polynomial of the best $L_{2}[-1,1]$ approximation of the function $g$ by polynomials of degree $2 N$. Hence by Theorem 6.3 from [10, Chap. 7] for any $r \in \mathbb{N}$ there exists a universal constant $C_{r}$ such that $\left\|g-g_{N}\right\|_{L_{2}[-1,1]} \leq C_{r} \omega_{r}\left(g, \frac{1}{2 N}\right)_{L_{2}[-1,1]}, 2 N \geq r$. We take $r=[l+3 / 2-\alpha]+1$. Then using the estimates (4.4) and (4.7) we obtain that

$$
\omega_{r}\left(g, \frac{1}{2 N}\right)_{L_{2}[-1,1]}=\frac{1}{\sqrt{x}} \omega_{r}\left(\widetilde{R}, \frac{x}{2 N}\right)_{L_{2}[-x, x]} \leq \frac{1}{\sqrt{x}} \omega_{r}\left(\widetilde{R}, \frac{x}{2 N}\right)_{L_{2}(\mathbb{R})} \leq \frac{C(q)}{\sqrt{x}}\left(\frac{x}{2 N}\right)^{l+3 / 2-\alpha},
$$

where the constant $C(q)$ depends neither on $x$ nor on $N$. To finish the proof of (4.11), note that $\left\|R(x, \cdot)-R_{N}(x, \cdot)\right\|_{L_{2}[0, x]}=\frac{1}{2}\left\|2 \widetilde{R}(x, \cdot)-R_{N}(x, \cdot)\right\|_{L_{2}[-x, x]}=\frac{1}{2} \sqrt{x}\left\|g-g_{N}\right\|_{L_{2}[-1,1]} \cdot$

To prove the estimate (4.12) we proceed as follows.

$$
\begin{aligned}
\left|\beta_{N}(x)\right| & =(4 N+1)\left|\int_{0}^{x} R(x, t) P_{2 N}\left(\frac{t}{x}\right) d t\right| \\
& =(4 N+1)\left|\int_{0}^{x}\left(R(x, t)-R_{N-1}(x, t)\right) P_{2 N}\left(\frac{t}{x}\right) d t\right| \\
& \leq(4 N+1)\left\|R(x, \cdot)-R_{N-1}(x, \cdot)\right\|_{L_{2}[0, x]} \cdot \sqrt{\frac{x}{4 N+1}} \leq \frac{C_{1} \sqrt{4 N+1} \cdot x^{l+2-\alpha}}{(N-1)^{l+3 / 2-\alpha}} \leq \frac{C_{2} x^{l+2-\alpha}}{(N-1)^{l+1-\alpha}},
\end{aligned}
$$

where we used the Cauchy-Schwarz inequality and the fact that $R_{N-1}$ is a polynomial in even powers of $t$ of degree lower than $2 N$, hence orthogonal to $P_{2 N}$.

Note that the estimates (4.11) and (4.12) do not depend on the smoothness of the potential q. In Subsection 9.1 we study the behavior of the coefficients $\beta_{n}$ numerically and observe that for some potentials the actual degree of $N$ in the decay rate of the coefficients $\beta_{n}$ is $2 l+3$, higher than $l+1-\alpha$ in (4.12). Below we give a proof for such improved decay rate requiring $q$ to be sufficiently smooth. Additionally, in Subsection 9.1 we observe that in the special case $l \in \mathbb{N}_{0}:=\mathbb{N} \cup\{0\}$ the coefficients $\beta_{n}$ decay much faster than for $l \notin \mathbb{N}_{0}$ and that the decay rate depends on the smoothness of the potential $q$. Below we present a theoretical justification of this phenomenon. We need the following lemma first. 
Lemma 4.4. Let $l \notin \mathbb{N}_{0}$ and $k \in \mathbb{N}_{0}$. Then there exists a constant $c_{k}$ such that the following inequalities hold

$$
\left|\int_{0}^{x}\left(1-\frac{t^{2}}{x^{2}}\right)^{l+k} P_{2 n}\left(\frac{t}{x}\right) d t\right| \leq \frac{c_{k} x}{n^{2 l+2 k+2}}, \quad n \geq l+k+2 .
$$

Proof. Using the Taylor series for the function $\left(1-\frac{t^{2}}{x^{2}}\right)^{l+k}$ and the formula [27, 2.17.1] we obtain that

$$
\begin{aligned}
\int_{0}^{x}\left(1-\frac{t^{2}}{x^{2}}\right)^{l+k} P_{2 n}\left(\frac{t}{x}\right) d t & =\sum_{m=n}^{\infty}(-1)^{m}\left(\begin{array}{c}
l+k \\
m
\end{array}\right) \frac{1}{x^{2 m}} \int_{0}^{x} t^{2 m} P_{2 n}\left(\frac{t}{x}\right) d t \\
& =\frac{x}{2 \Gamma(-l-k)} \sum_{m=n}^{\infty} \frac{\Gamma(m-l-k) \Gamma(m+1 / 2)}{\Gamma(m-n+1) \Gamma(m+n+3 / 2)}
\end{aligned}
$$

Denote the terms of the last series as $a_{m}$. We have that $a_{m} \geq 0$, and one can check by a simple verification that $\frac{a_{m+1}}{a_{m}} \geq 1$ for $m \leq \frac{n^{2}-3 / 2}{l+k+2}$ and $\frac{a_{m+1}}{a_{m}}<1$ for $m>\frac{n^{2}-3 / 2}{l+k+2} \geq n-1$. Using the asymptotic formula [1, (6.1.40)], $\log \Gamma(z)=\left(z-\frac{1}{2}\right) \log z-z+\frac{1}{2} \log 2 \pi+O\left(\frac{1}{z}\right)$, we can check that

$$
\log a_{m}=-(l+k+2) \log m+O\left(\frac{1}{m}\right), \quad m \geq n,
$$

with the uniform bound of the error term. Hence $a_{m} \leq \frac{c}{m^{k+l+2}}, m \geq n$ and

$$
\begin{aligned}
\sum_{m=n}^{\infty} a_{m} & \leq \sum_{m=n}^{\left[\frac{n^{2}-3 / 2}{l+k+2}+1\right]} a_{\left[\frac{n^{2}-3 / 2}{l+k+2}+1\right]}+\sum_{m=\left[\frac{n^{2}-3 / 2}{l+k+2}+2\right]}^{\infty} \frac{c}{m^{l+k+2}} \\
& \leq \frac{n^{2}}{l+k+2} \frac{c(l+k+2)^{l+k+2}}{\left(n^{2}-3 / 2\right)^{l+k+2}}+\frac{c}{l+k+1} \frac{(l+k+2)^{l+k+1}}{\left(n^{2}-3 / 2\right)^{l+k+1}}
\end{aligned}
$$

finishing the proof.

Proposition 4.5. Suppose that $l \in \mathbb{N}$ and $q \in C^{2 p-1}[0, b]$ for some $p \in \mathbb{N}$. Then the function $\widetilde{R}$ from Proposition 4.1 satisfies $\widetilde{R} \in \operatorname{Lip}_{l+1+p-\varepsilon}([-x, x])$ for any $\varepsilon>0$ and $\widetilde{R} \in \operatorname{Lip}_{l+p+3 / 2}^{*}([-x, x], 2)$. Moreover, there exist constants $c_{1}$ and $c_{2}$, dependent on $q, l$ and $p$, such that for any $x>0$ the inequalities hold

$$
\left\|R(x, \cdot)-R_{N}(x, \cdot)\right\|_{L_{2}[0, x]} \leq \frac{c_{1} x^{l+p+3 / 2}}{N^{l+p+3 / 2}}, \quad 2 N \geq[l+p+5 / 2]
$$

and

$$
\left|\beta_{N}(x)\right| \leq \frac{c_{2} x^{l+p+2}}{(N-1)^{l+p+1}}, \quad 2 N \geq[l+p+9 / 2] .
$$

Suppose that $l \notin \mathbb{N}_{0}$ and $q \in C^{2 p-1}[0, b]$. Then there exist constants $c_{3}$ and $c_{4}$ such that for any $x>0$ the inequalities hold

$$
\left\|R(x, \cdot)-R_{N}(x, \cdot)\right\|_{L_{2}[0, x]} \leq \frac{c_{3} x^{l+3 / 2}}{N^{r}}, \quad N \geq[l+p+3]
$$

and

$$
\left|\beta_{N}(x)\right| \leq \frac{c_{4} x^{l+2}}{N^{r}}, \quad N \geq[l+p+3]
$$

where $r=\min \{l+p+1,2 l+3\}$. 
Proof. In [1] the following asymptotic expansion for $u_{l}(\omega, x)$ was obtained

$$
u_{l}(\omega, x)=\sum_{k=0}^{m} A_{k}(x) \frac{\sqrt{x} J_{l+k+1 / 2}(\omega x)}{\omega^{l+k+1 / 2}}+\mathcal{R}_{m}(\omega, x) .
$$

The coefficients $A_{k}$ are defined as follows. Consider the operators $H_{p}, p=1,2, \ldots$, acting as

$$
\left(H_{p} f\right)(x)= \begin{cases}\frac{1}{x^{p}} \int_{0}^{x} t^{p-1} f(t) d t, & \text { if } x \neq 0, \\ \frac{1}{p} f(0), & \text { if } x=0 .\end{cases}
$$

One can verify that if $f \in C^{(r)}[0, b]$ then $H_{p} f \in C^{(r)}[0, b]$ and $\left(H_{p} f\right)^{(r)}=H_{p+r}\left(f^{(r)}\right), r=0,1,2, \ldots$ Let $A_{k}(x)=: x^{k} B_{k}(x)$. Then the functions $B_{k}$ satisfy the following recursive relations

$$
B_{0}=2^{l+1 / 2} \Gamma(l+3 / 2)
$$

(with such choice of $B_{0}$ the first term of (4.18) coincides with $d(\omega) b_{l}(\omega x)$ ) and

$$
B_{k+1}=-\frac{1}{2} H_{k+1}\left[B_{k}^{\prime \prime}-2 l H_{1} B_{k}^{\prime \prime}-q B_{k}\right] .
$$

Moreover, their derivatives satisfy the equalities

$$
B_{k+1}^{(j)}=-\frac{1}{2} H_{k+j+1}\left[B_{k}^{(j+2)}-2 l H_{j+1} B_{k}^{(j+2)}-\left(q B_{k}\right)^{(j)}\right], \quad j \in \mathbb{N} .
$$

For $\omega \in \mathbb{R},|\omega| \geq 1$ the remainder $\mathcal{R}_{m}$ satisfies the inequality

$$
\left|\mathcal{R}_{m}(\omega, x)\right| \leq \frac{c(l, m)}{|\omega|^{l+m+2}} \int_{0}^{x}\left|\left(t^{m+1} B_{m+1}(t)\right)^{\prime}\right| d t
$$

and, as can be seen from (4.18), $\mathcal{R}_{m}(\omega, x)$ is an even entire function of the complex variable $\omega$.

Observe that the condition $q \in C^{2 k+j}[0, b]$ is sufficient for the $j$-th derivative of the function $B_{k+1}$ to be well defined and bounded on $[0, b]$ via the formulas (4.19)-(4.21). Hence it is sufficient to have $q \in C^{2 p-2}[0, b]$ to obtain the coefficients $A_{0}, \ldots, A_{p}$. As for the expression $\left(x^{p+1} B_{p+1}(x)\right)^{\prime}$, we show that the condition $q \in C^{2 p-1}[0, b]$ is sufficient, one does not need to ask for $q$ to be $2 p+1$ times differentiable. Indeed, it follows from (4.20) that

$$
\left(x^{p+1} B_{p+1}\right)^{\prime}=-\frac{1}{2}\left(\left(x^{p} B_{p}\right)^{\prime \prime}-2(p+l) x^{p-1} B_{p}^{\prime}-p(p-1) x^{p-2} B_{p}+2 l x^{p-1} B_{p}^{\prime}(0)-x^{p} q B_{p}\right),
$$

and the last four terms are well defined whenever $q \in C^{2 p-1}[0, b]$, as was explained above. For the first term we consider two cases. If $p=1$ then $\left(x B_{1}\right)^{\prime \prime}=-\frac{B_{0}}{2} q^{\prime}(x)$, and for $p>1$ using (4.20) we have that the expression

$$
\left(x^{p} B_{p}\right)^{\prime \prime}=-\frac{1}{2}\left(x^{p-1} B_{p-1}^{\prime \prime}-2 l x^{p-2} B_{p-1}^{\prime}+2 l x^{p-2} B_{p-1}^{\prime}(0)-x^{p-1} q B_{p-1}\right)^{\prime}
$$

is well defined whenever $q \in C^{2 p-1}[0, b]$.

Applying the Paley-Wiener theorem as in the proof of Proposition 4.1 we obtain that

$$
\mathcal{R}_{p}(\omega, x)=\int_{-x}^{x} \hat{\mathcal{R}}(x, t) e^{i \omega t} d t
$$


where the function $\hat{\mathcal{R}}(x, \cdot) \in W^{l+p+3 / 2-\varepsilon}(\mathbb{R}) \cap \operatorname{Lip}_{l+p+1-\varepsilon}(\mathbb{R}) \cap \operatorname{Lip}_{l+p+3 / 2}^{*}(\mathbb{R}, 2)$ and $\operatorname{supp} \hat{\mathcal{R}}(x, \cdot) \subset$ $[-x, x]$. From the formula [23, (5.10.2)], $J_{\nu}(z)=\frac{(z / 2)^{\nu}}{\sqrt{\pi} \Gamma(\nu+1 / 2)} \int_{-1}^{1}\left(1-t^{2}\right)^{\nu-1 / 2} \cos (z t) d t$ we have

$$
\frac{\sqrt{x} J_{l+k+1 / 2}(\omega x)}{\omega^{l+k+1 / 2}}=\frac{x^{l+k}}{\sqrt{\pi} 2^{l+k+1 / 2} \Gamma(l+k+1)} \int_{-x}^{x}\left(1-\frac{z^{2}}{x^{2}}\right)^{l+k} e^{i \omega z} d z .
$$

Comparing the expressions (4.5), (4.18), (4.22) and (4.23) one can see that

$$
\widetilde{R}(x, t)-\hat{\mathcal{R}}(x, t)=\sum_{k=1}^{p} \frac{A_{k}(x) x^{l+k}}{\sqrt{\pi} 2^{l+k+1 / 2} \Gamma(l+k+1)} \cdot\left(1-\frac{t^{2}}{x^{2}}\right)^{l+k}, \quad-x \leq t \leq x .
$$

Suppose that $l \in \mathbb{N}_{0}$. Then for every fixed $x$, the right-hand side of (4.24) is a polynomial in $t$, i.e., a $C^{\infty}$-function implying that the smoothness of $\widetilde{R}(x, \cdot)$ coincides with the smoothness of $\hat{\mathcal{R}}(x, \cdot)$ for $t \in[-x, x]$. Now one can obtain all remaining statements of the proposition similarly to the proof of Theorem 4.3 .

Suppose that $l \notin \mathbb{N}_{0}$. Consider the Fourier-Legendre series for $\hat{\mathcal{R}}$,

$$
\hat{\mathcal{R}}(x, t)=\frac{1}{2} \sum_{n=0}^{\infty} \frac{\hat{\beta}_{n}(x)}{x} P_{2 n}\left(\frac{t}{x}\right) .
$$

Similarly to the proof of Theorem 4.3 we have that $\left|\hat{\beta}_{N}(x)\right| \leq c x^{l+p+2}(N-1)^{-l-p-1}$ when $2 N \geq$ $[l+p+9 / 2]$. The Fourier-Legendre coefficients for the right-hand side of (4.24) can be estimated using Lemma 4.4. Combining the estimates one obtains (4.17). The difference $R-R_{N}$ can be estimated using (4.17) and the orthogonality of the Legendre polynomials. One has

$$
\left\|R(x, \cdot)-R_{N}(x, \cdot)\right\|_{L_{2}[0, x]}^{2}=\sum_{n=N+1}^{\infty} \frac{\left|\beta_{n}(x)\right|^{2}}{x^{2}} \cdot \frac{x}{4 n+1} \leq \frac{c_{4}^{2} x^{2 l+3}}{2 r N^{2 r}} .
$$

Remark 4.6. The estimates (4.16) and (4.17) present a saturation phenomenon, the exponent $r$ can not exceed $2 l+3$ whatever smooth the potential $q$ is. It is not a technical restriction, but an intrinsic property of the proposed representation. Indeed, one can deduce similarly to the proof of Lemma 4.4 that the order $2 n+2 k+2$ in (4.13) can not be improved. Hence, whenever one has $A_{1}(x)=\frac{A_{0}}{2} Q(x) \neq 0$ in (4.24), the decay rate order of the coefficients $\beta_{n}(x)$ for sufficiently smooth potential $q$ is determined by the first term in (4.24) and can not exceed $2 l+3$.

Remark 4.7. Using additional terms from the asymptotic expansion (4.18) together with (4.22) in comparison with representation (4.1) may result in a modification of the method proposed in this paper allowing one to obtain an improved convergence rate. We leave the detailed analysis for a separate paper.

\section{Representation of the regular solution}

Here we prove the main result of the present work.

Theorem 5.1. Under the conditions of Theorem 4.3, the regular solution $u_{l}(\omega, x)$ of (3.1) satisfying the asymptotic relation $u_{l}(\omega, x) \sim x^{l+1}$ when $x \rightarrow 0$ has the form

$$
u_{l}(\omega, x)=d(\omega) b_{l}(\omega x)+\sum_{n=0}^{\infty}(-1)^{n} \beta_{n}(x) j_{2 n}(\omega x)
$$


where $\beta_{n}$ are defined by (4.9) and $j_{2 n}$ stands for the spherical Bessel function of the first kind of order $2 n$, the series converges uniformly with respect to $x$ on $[0, b]$ and converges uniformly with respect to $\omega$ on any finite subset of the complex plane of the variable $\omega$.

For the approximate solution

$$
u_{l ; N}(\omega, x)=d(\omega) b_{l}(\omega x)+\sum_{n=0}^{N}(-1)^{n} \beta_{n}(x) j_{2 n}(\omega x)
$$

the following estimate holds

$$
\left|u_{l}(\omega, x)-u_{l ; N}(\omega, x)\right| \leq \sqrt{x} \varepsilon_{N}(x)
$$

for any $\omega \in \mathbb{R}, \omega \neq 0$, and

$$
\left|u_{l}(\omega, x)-u_{l ; N}(\omega, x)\right| \leq\left(\frac{\sinh (2 C x)}{2 C}\right)^{1 / 2} \varepsilon_{N}(x)
$$

for any $\omega \in \mathbb{C}, \omega \neq 0$ belonging to the strip $|\operatorname{Im} \omega| \leq C, C \geq 0$, where $\varepsilon_{N}$ is a sufficiently small nonnegative function such that $\left\|R(x, \cdot)-R_{N}(x, \cdot)\right\|_{L_{2}[0, x]} \leq \varepsilon_{N}(x)$ which exists due to Theorem 4.3 (an estimate for $\varepsilon_{N}(x)$ is presented in (4.11)).

Moreover, for each fixed $x$ and $\omega$ the convergence rate of $u_{l ; N}(\omega, x)$ to $u_{l}(\omega, x)$ is exponential. To be more precise, let $x>0$ be fixed and $\omega \in \mathbb{C}$ satisfy $|\omega| \leq \omega_{0}$. Then for all $N>\omega_{0} x / 2$ one has

$$
\left|u_{l}(\omega, x)-u_{l ; N}(\omega, x)\right| \leq \frac{c x e^{|\operatorname{Im} \omega| x}}{N^{l+1-\alpha}} \cdot \frac{1}{(2 N+2) !} \cdot\left|\frac{\omega_{0} x}{2}\right|^{2 N+2}
$$

where $c$ is a constant depending on $q$ and $l$ only and $\alpha$ is the constant from the condition (4.2).

Proof. Consider the solution (4.1). For the integral from (4.1) using formula 2.17.7 from [27, p. 433] we obtain

$$
\int_{0}^{x} R(x, t) \cos \omega t d t=\sum_{n=0}^{\infty} \frac{\beta_{n}(x)}{x} \int_{0}^{x} P_{2 n}\left(\frac{t}{x}\right) \cos \omega t d t=\sum_{n=0}^{\infty}(-1)^{n} \beta_{n}(x) j_{2 n}(\omega x) .
$$

Using the Cauchy-Schwarz inequality we obtain that

$$
\begin{aligned}
\left|u_{l}(\omega ; x)-u_{l ; N}(\omega ; x)\right| & =\left|\int_{0}^{x}\left(R(x, t)-R_{N}(x, t)\right) \cos \omega t d t\right| \\
& \leq\left\|R(x, \cdot)-R_{N}(x, \cdot)\right\|_{L_{2}[0, x]} \cdot\left(\int_{0}^{x}\left|\cos ^{2} \omega t\right| d t\right)^{1 / 2} .
\end{aligned}
$$

The estimates (5.3) and (5.4) follow immediately from this inequality by taking into account that for complex $\omega$ one has

$$
\int_{0}^{x}\left|\cos ^{2} \omega t\right| d t=\frac{1}{2} \int_{0}^{x}(\cosh (2 \operatorname{Im} \omega t)+1) d t=\frac{\sinh (2|\operatorname{Im} \omega| x)}{4|\operatorname{Im} \omega|}+\frac{x}{2} \leq \frac{\sinh (2|\operatorname{Im} \omega| x)}{2|\operatorname{Im} \omega|}
$$

and that the function $\sinh t / t$ is monotone increasing for $t>0$.

The uniform convergence with respect to $x$ follows from the estimate (4.11) for $\varepsilon_{N}(x)$.

The estimate (5.5) can be obtained using the estimate (4.12) similarly to the proof of [18, Proposition 4.4]. 
Remark 5.2. Note that the function $u_{l}(\omega, x)$, as a function of $\omega \in \mathbb{R}$, is bounded by $C\left(\frac{x}{b+|\omega| x}\right)^{l+1}$, see [15], i.e., is decaying fast when $\omega \rightarrow \infty$, especially for large values of the parameter l. Hence, though the uniform estimate (5.3) holds for all $\omega \in \mathbb{R}$, for a large $l$ it is practical only for $\omega$ close to zero. For small values of the parameter $l$ one obtains a quite large region (sufficient to compute hundreds of eigenvalues, for example), while for large values of $l$ the usable region of $\omega$ 's is much smaller (suitable to compute a dozen of eigenvalues at most).

\section{Recurrent equations for $\beta_{n}$}

Let us substitute the solution (5.1) into equation (3.1). Definition (4.9) and estimate (4.12) together with the inequality [1, (9.1.62)], $\left|j_{n}(x)\right| \leq \sqrt{\pi}\left|\frac{x}{2}\right|^{n} \frac{1}{\Gamma(n+3 / 2)}, x \in \mathbb{R}$ justify the possibility to differentiate the series (5.1) termwise. Consider

$$
L\left[\beta_{n}(x) j_{2 n}(\omega x)\right]=\beta_{n}(x) L\left[j_{2 n}(\omega x)\right]-\beta_{n}^{\prime \prime}(x) j_{2 n}(\omega x)-2 \beta_{n}^{\prime}(x) j_{2 n}^{\prime}(\omega x)
$$

where the prime means the derivative with respect to $x$. The following formulas are used

$$
\frac{d j_{k}(z)}{d z}=-j_{k+1}(z)+\frac{k}{z} j_{k}(z) \quad \text { and } \quad \frac{d j_{k}(z)}{d z}=j_{k-1}(z)-\frac{k+1}{z} j_{k}(z) .
$$

Thus,

$$
j_{2 n}^{\prime}(\omega x)=-\omega\left(j_{2 n+1}(\omega x)-\frac{2 n}{\omega x} j_{2 n}(\omega x)\right)
$$

and

$$
j_{2 n}^{\prime \prime}(\omega x)=-j_{2 n}(\omega x)\left(\omega^{2}-\frac{2 n(2 n-1)}{x^{2}}\right)+\frac{2 \omega}{x} j_{2 n+1}(\omega x)
$$

Hence

$$
L\left[j_{2 n}(\omega x)\right]=j_{2 n}(\omega x)\left(\omega^{2}-\frac{2 n(2 n-1)}{x^{2}}\right)-\frac{2 \omega}{x} j_{2 n+1}(\omega x)+q_{l}(x) j_{2 n}(\omega x)
$$

where $q_{l}(x):=\left(\frac{l(l+1)}{x^{2}}+q(x)\right)$ and thus,

$$
\begin{aligned}
& 0=L\left[u_{l}(\omega, x)\right]-\omega^{2} u_{l}(\omega, x)=d(\omega) q(x) b_{l}(\omega x) \\
& +\sum_{n=0}^{\infty}(-1)^{n}\left[\beta_{n}(x)\left(j_{2 n}(\omega x)\left(q_{l}(x)-\frac{2 n(2 n-1)}{x^{2}}\right)-\frac{2 \omega}{x} j_{2 n+1}(\omega x)\right)\right. \\
& \left.\quad-\beta_{n}^{\prime \prime}(x) j_{2 n}(\omega x)+2 \beta_{n}^{\prime}(x)\left(\omega j_{2 n+1}(\omega x)-\frac{2 n}{x} j_{2 n}(\omega x)\right)\right] .
\end{aligned}
$$

We obtain the equality, which after applying the formula

$$
j_{2 n}(\omega x)=\frac{\omega x}{4 n+1}\left(j_{2 n-1}(\omega x)+j_{2 n+1}(\omega x)\right)
$$

can be written in the form

$$
\frac{d(\omega) q(x) b_{l}(\omega x)}{\omega x}-q_{l}(x) j_{-1}(\omega x)=\sum_{n=1}^{\infty} \alpha_{n}(x) j_{2 n-1}(\omega x)
$$


with

$$
\begin{aligned}
\alpha_{n}(x):= & (-1)^{n}\left(\frac{1}{4 n+1}\left(\beta_{n}^{\prime \prime}(x)+\frac{4 n}{x} \beta_{n}^{\prime}(x)+\left(\frac{2 n(2 n-1)}{x^{2}}-q_{l}(x)\right) \beta_{n}(x)\right)\right. \\
& -\frac{1}{4 n-3}\left(\beta_{n-1}^{\prime \prime}(x)+\frac{4(n-1)}{x} \beta_{n-1}^{\prime}(x)+\left(\frac{2(n-1)(2(n-1)-1)}{x^{2}}-q_{l}(x)\right) \beta_{n-1}(x)\right) \\
& \left.+2\left(\frac{\beta_{n-1}^{\prime}(x)}{x}-\frac{\beta_{n-1}(x)}{x^{2}}\right)\right) .
\end{aligned}
$$

Multiplying equality (6.2) by $j_{2 m-1}(\omega x), m=1,2, \ldots$, integrating with respect to $\omega$ from 0 to $\infty$ and using the integrals

$$
\int_{0}^{\infty} j_{2 n-1}(\omega x) j_{2 m-1}(\omega x) d \omega= \begin{cases}0, & m \neq n \\ \frac{\pi}{2 x(4 m-1)}, & m=n\end{cases}
$$

for $n, m \in \mathbb{Z}$ with $m+n-1>-1 / 2$ (c.f., [1, Formula 11.4.6]) we obtain

$$
\frac{\pi \alpha_{n}(x)}{2(4 n-1)}=q(x) \int_{0}^{\infty} \frac{d(\omega) b_{l}(\omega x)}{\omega} j_{2 n-1}(\omega x) d \omega .
$$

Consider the integral

$$
\begin{aligned}
\int_{0}^{\infty} \frac{b_{l}(\omega x)}{\omega^{l+2}} j_{2 n-1}(\omega x) d \omega & =\sqrt{\frac{\pi}{2}} \int_{0}^{\infty} \frac{J_{l+1 / 2}(\omega x) J_{2 n-1 / 2}(\omega x)}{\omega^{l+2}} d \omega \\
& =\sqrt{\frac{\pi}{2}} \frac{\left(\frac{x}{2}\right)^{l+1} \Gamma(l+2) \Gamma(n-1 / 2)}{2 \Gamma(l-n+2) \Gamma(n+1) \Gamma(n+l+3 / 2)}
\end{aligned}
$$

where formula (1) from [34, Sect. 13.41] was used. Hence

$$
\int_{0}^{\infty} \frac{d(\omega) b_{l}(\omega x)}{\omega} j_{2 n-1}(\omega x) d \omega=\frac{x^{l+1} \sqrt{\pi} \Gamma(l+2) \Gamma(l+3 / 2) \Gamma(n-1 / 2)}{4 \Gamma(l-n+2) \Gamma(n+1) \Gamma(n+l+3 / 2)} .
$$

Thus,

$$
\alpha_{n}(x)=B_{n} q(x) x^{l+1}, \quad \text { with } B_{n}:=\frac{(4 n-1) \Gamma(l+2) \Gamma(l+3 / 2) \Gamma(n-1 / 2)}{2 \sqrt{\pi} \Gamma(l-n+2) \Gamma(n+1) \Gamma(n+l+3 / 2)} .
$$

It is easy to see (c.f., [18] and ([6.3) $)$ that this equation can be written in the following form

$$
\frac{1}{(4 n+1) x^{2 n}} L\left[x^{2 n} \beta_{n}\right]=\frac{x^{2 n-1}}{4 n-3} L\left[\frac{\beta_{n-1}}{x^{2 n-1}}\right]-(-1)^{n} B_{n} q(x) x^{l+1} .
$$

Thus, we obtained a sequence of equations satisfied by the coefficients $\beta_{n}$.

A solution $u$ of an equation

$$
L u=h
$$

can be easily obtained using the Pólya factorization of $L, L u=-\frac{1}{u_{0}} \partial u_{0}^{2} \partial \frac{u}{u_{0}}$, where $\partial$ denotes the derivative with respect to $x$ and $u_{0}$ is the same solution as in Section 3 . The function

$$
u(x)=-u_{0}(x) \int_{0}^{x} \frac{1}{u_{0}^{2}(t)} \int_{0}^{t} u_{0}(s) h(s) d s d t
$$


is a solution of (6.5) provided, e.g., that $|h(x)| \leq C x^{l-1+\varepsilon}$ in a neighborhood of zero for some positive $C$ and $\varepsilon$, see [6] for further details. Note also that the expression (6.6) gives the unique solution of (6.5) satisfying $u(x)=o\left(x^{l+1}\right), x \rightarrow 0$.

One can see from (3.6) that the functions $\varphi_{n}$ satisfy $\left|\varphi_{n}(x)\right| \leq c_{n, 1} x^{2 n+l+1},\left|\varphi_{n}^{\prime}(x)\right| \leq c_{n, 2} x^{2 n+l}$ and $\left|\varphi_{n}^{\prime \prime}(x)\right| \leq c_{n, 3} x^{2 n+l-1}, n \geq 0$, for some constants $c_{n, i}$. Hence it follows from (4.9) (c.f., (4.12)) that $\left|\beta_{n}(x)\right| \leq c_{n, 4} x^{l+1},\left|\beta_{n}^{\prime}(x)\right| \leq c_{n, 5} x^{l}$ and $\left|\beta_{n}^{\prime \prime}(x)\right| \leq c_{n, 6} x^{l-1}, x>0$. These estimates justify that the expression (6.6) can be used to construct the functions $\beta_{n}, n \geq 1$ from equations (6.4). One starts with

$$
\beta_{0}(x)=u_{0}(x)-x^{l+1}
$$

and define for $n \geq 1$

$$
\beta_{n}(x)=-\frac{(4 n+1) u_{0}(x)}{x^{2 n}} \int_{0}^{x} \frac{1}{u_{0}^{2}(t)} \int_{0}^{t} u_{0}(s)\left[\frac{s^{4 n-1}}{4 n-3} L\left(\frac{\beta_{n-1}}{s^{2 n-1}}\right)-(-1)^{n} B_{n} q(s) s^{2 n+l+1}\right] d s d t .
$$

Note that for an integer $l$ the second term under the integral sign is equal to zero for $n \geq l+2$. To eliminate the first and second derivatives of $\beta_{n-1}$ resulting from the term $L\left(\frac{\beta_{n-1}}{s^{2 n-1}}\right)$, one may apply the integration by parts and obtain (similarly to [18]) the following recurrent formulas.

$$
\begin{array}{ll}
\eta_{n}(x)=\int_{0}^{x}\left(t u_{0}^{\prime}(t)+(2 n-1) u_{0}(t)\right) t^{2 n-2} \beta_{n-1}(t) d t, & \kappa_{n}(x)=\int_{0}^{x} u_{0}(t) q(t) t^{2 n+l+1} d t \\
\theta_{n}(x)=\int_{0}^{x} \frac{1}{u_{0}^{2}(t)}\left(\eta_{n}(t)-t^{2 n-1} \beta_{n-1}(t) u_{0}(t)\right) d t, & \mu_{n}(x)=\int_{0}^{x} \frac{\kappa_{n}(t)}{u_{0}^{2}(t)} d t, \quad n \geq 1,
\end{array}
$$

and finally

$$
\beta_{n}(x)=\frac{4 n+1}{4 n-3}\left[\beta_{n-1}(x)+\frac{u_{0}(x)}{x^{2 n}}\left[2(4 n-1) \theta_{n}(x)+(-1)^{n}(4 n-3) B_{n} \mu_{n}(x)\right]\right] .
$$

\section{Representation of the derivative of the regular solution}

In order to obtain a series expansion for $u_{l}^{\prime}(\omega, x)$ uniformly convergent with respect to $\omega$ (here and below prime means the derivative with respect to $x$ ) we return to (4.1) from which

$$
u_{l}^{\prime}(\omega, x)=\omega d(\omega) b_{l}^{\prime}(\omega x)+\int_{0}^{x} R_{1}(x, t) \cos \omega t d t
$$

with the subindex " 1 " denoting the partial derivative with respect to the first argument. Here we took into account that by construction $R(x, x) \equiv 0$ (see (3.12) and (3.10), also Proposition 4.1).

We have

$$
\begin{aligned}
R_{1}(x, s) & =A \frac{\partial}{\partial x} \int_{s}^{x} V(x, t)\left(t-\frac{s^{2}}{t}\right)^{l} d t \\
& =A\left(\frac{Q(x)}{2} x^{-l}\left(x^{2}-s^{2}\right)^{l}+\int_{s}^{x} V_{1}(x, t)\left(t-\frac{s^{2}}{t}\right)^{l} d t\right)
\end{aligned}
$$

where

$$
A:=\frac{2 \Gamma(l+3 / 2)}{\sqrt{\pi} \Gamma(l+1)}
$$

To obtain (17.2) we used (3.2). 
Denote

$$
R^{(1)}(x, s):=\frac{A Q(x)}{2} x^{-l}\left(x^{2}-s^{2}\right)^{l} \quad \text { and } \quad R^{(2)}(x, s):=A \int_{s}^{x} V_{1}(x, t)\left(t-\frac{s^{2}}{t}\right)^{l} d t .
$$

Notice that for $-1 / 2 \leq l<0$ the function $R^{(1)}$ has a singularity when $x=s$. In order to calculate $u_{l}^{\prime}(\omega, x)$ from (7.1) we split the integral into two parts

$$
\int_{0}^{x} R_{1}(x, t) \cos \omega t d t=\int_{0}^{x} R^{(1)}(x, t) \cos \omega t d t+\int_{0}^{x} R^{(2)}(x, t) \cos \omega t d t .
$$

Consider

$$
\int_{0}^{x} R^{(1)}(x, t) \cos \omega t d t=\frac{A Q(x)}{2} x^{-l} \int_{0}^{x}\left(x^{2}-s^{2}\right)^{l} \cos \omega t d t .
$$

Due to Remark 2.2 we obtain that

$$
\int_{0}^{x} R^{(1)}(x, t) \cos \omega t d t=\frac{d(\omega)}{2} Q(x) b_{l}(\omega x) .
$$

Thus,

$$
u_{l}^{\prime}(\omega, x)=d(\omega)\left(\omega b_{l}^{\prime}(\omega x)+\frac{Q(x)}{2} b_{l}(\omega x)\right)+\int_{0}^{x} R^{(2)}(x, t) \cos \omega t d t .
$$

The article [33] does not provide sufficient details on the behavior of the derivative $V_{1}(x, t)$ near $t=0$. As a result, the definition (7.3) is not quite helpful for studying the integral kernel $R^{(2)}$, even the integrability of $R^{(2)}(x, t)$ near $t=0$ necessary for the representation (7.4) to be well defined goes under the question. Below we provide a different proof of the representation (17.4) based on the Paley-Wiener theorem and similar to that of Proposition 4.1.

Theorem 7.1. Assume additionally to the conditions of Theorem 3.2 that $q \in C^{1}[0, b]$. Let $x>0$ be fixed. Then there exists an even, compactly supported on $[-x, x]$ function $\widetilde{R}^{(2)}(x, t)$ such that $\widetilde{R}^{(2)}(x, \cdot) \in W_{2}^{l+3 / 2-\varepsilon}(\mathbb{R}) \cap \operatorname{Lip}_{l+1-\varepsilon}(\mathbb{R}) \cap \operatorname{Lip}_{l+3 / 2}^{*}(\mathbb{R})$ for any small $\varepsilon>0$ and the representation (7.4) holds with the function $R^{(2)}$ satisfying $R^{(2)}(x, t)=2 \widetilde{R}^{(2)}(x, t), 0 \leq t \leq x$.

The kernel $R^{(2)}$ from (7.3) admits the following representation

$$
R^{(2)}(x, t)=\sum_{n=0}^{\infty} \frac{\gamma_{n}(x)}{x} P_{2 n}\left(\frac{t}{x}\right)
$$

where

$$
\gamma_{n}(x)=(4 n+1) \sum_{k=0}^{n} \frac{l_{2 k, 2 n}}{x^{2 k}}\left(\varphi_{k}^{\prime}(x)-c_{k, l}\left((2 k+l+1) x^{2 k+l}+\frac{Q(x)}{2} x^{2 k+l+1}\right)\right) .
$$

The convergence of the series in (7.5) is the same as stated in Theorem 4.3 for the series (4.8).

Denote

$$
R_{N}^{(2)}(x, t):=\sum_{n=0}^{N} \frac{\gamma_{n}(x)}{x} P_{2 n}\left(\frac{t}{x}\right)
$$

Then there exist constants $C_{1}$ and $C_{2}$, dependent on $q$ and $l$ and independent of $x$ and $N$, such that for any $x>0$ the inequalities hold

$$
\left\|R^{(2)}(x, \cdot)-R_{N}^{(2)}(x, \cdot)\right\|_{L_{2}[0, x]} \leq \frac{C_{1} x^{l+3 / 2}}{N^{l+3 / 2}}, \quad 2 N \geq[l+5 / 2]
$$


and

$$
\left|\gamma_{N}(x)\right| \leq \frac{C_{2} x^{l+2}}{(N-1)^{l+1}}, \quad 2 N \geq[l+9 / 2] .
$$

Let $q \in C^{2 p+1}[0, b]$ with $p \geq 1$. If $l \in \mathbb{N}_{0}$, then the smoothness of $\widetilde{R}^{(2)}$ and the order of $N$ in the estimates (7.7) and (7.8) can be increased by $p$; if $l \notin \mathbb{N}_{0}$, then the order of $N$ in the estimates (7.7) and (7.8) can be increased to $r=\min \{l+p+1,2 l+3\}$, c.f., Proposition 4.5

Proof. Consider the asymptotic expansion (4.18) with $m=1$. According to [11] the remainder $\mathcal{R}_{1}(\omega, x)$ satisfies the integral equation

$$
\mathcal{R}_{1}(\omega, x)=\int_{0}^{x} G_{l}(\omega, x, t)\left(q(t) \mathcal{R}_{1}(\omega, t)+2 A_{2}^{\prime}(t) \frac{\sqrt{t} J_{l+3 / 2}(\omega t)}{\omega^{l+3 / 2}}\right) d t
$$

where (c.f., [15])

$$
G_{l}(\omega, x, t)=-\frac{\pi}{2} \sqrt{x t}\left(J_{l+1 / 2}(\omega x) Y_{l+1 / 2}(\omega t)-J_{l+1 / 2}(\omega t) Y_{l+1 / 2}(\omega x)\right)
$$

and

$$
A_{2}^{\prime}(x)=-\frac{1}{2}\left(A_{1}^{\prime \prime}(x)-\frac{2(l+1)}{x} A_{1}^{\prime}(x)+\left(\frac{2(l+1)}{x^{2}}-q(x)\right) A_{1}(x)\right) .
$$

Observe that $A_{1}(x)=\frac{A_{0}}{2} Q(x)$, hence

$$
\begin{aligned}
A_{2}^{\prime}(x) & =-\frac{A_{0}}{2}\left(\frac{q^{\prime}(x)}{2}-\frac{l+1}{x} q(x)+\frac{l+1}{x^{2}} Q(x)-\frac{q(x) Q(x)}{2}\right) \\
& =-\frac{A_{0}}{2}\left(\frac{q^{\prime}(x)}{2}-\frac{l+1}{x^{2}} \int_{0}^{x} t q^{\prime}(t) d t-\frac{q(x) Q(x)}{2}\right) .
\end{aligned}
$$

Since $q \in C^{1}[0, b], A_{2}^{\prime} \in C(0, b]$ and can be extended to a $C[0, b]$ function by continuity. Denote $A_{2}:=\max _{[0, b]}\left|A_{2}^{\prime}(x)\right|$.

The derivative $\mathcal{R}_{1, x}:=\frac{\partial}{\partial x} \mathcal{R}_{1}$ satisfies

$$
\mathcal{R}_{1, x}(\omega, x)=\int_{0}^{x} \frac{\partial}{\partial x} G_{l}(\omega, x, t)\left(q(t) \mathcal{R}_{1}(\omega, t)+2 A_{2}^{\prime}(t) \frac{\sqrt{t} J_{l+3 / 2}(\omega t)}{\omega^{l+3 / 2}}\right) d t .
$$

Recall the following estimates for the functions $G_{l}$ and $J_{l+3 / 2}$ (see [15, Appendix 1]), here $\omega \in \mathbb{R}$ and $t \leq x$.

$$
\begin{aligned}
\left|G_{l}(\omega, x, t)\right| & \leq C\left(\frac{x}{b+|\omega| x}\right)^{l+1}\left(\frac{b+|\omega| t}{t}\right)^{l} \theta(t), \\
\left|\frac{\partial}{\partial x} G_{l}(\omega, x, t)\right| & \leq C\left(\frac{x}{b+|\omega| x}\right)^{l}\left(\frac{b+|\omega| t}{t}\right)^{l} \theta(t),
\end{aligned}
$$

where $\theta(t)=1$ for $l>-1 / 2$ and $\theta(t)=1-\log (t / b)$ for $l=-1 / 2$, and

$$
\sqrt{x}\left|J_{l+3 / 2}(\omega x)\right| \leq C_{1} \sqrt{\frac{2}{\pi}} \omega^{l+3 / 2}\left(\frac{x}{b+|\omega| x}\right)^{l+2} .
$$

Applying the successive approximations method similarly to [26, Chap. 6, §10]) one easily obtains that

$$
\left|\mathcal{R}_{1}(\omega, x)\right| \leq \frac{\widetilde{C}}{|\omega|^{2}}\left(\frac{x}{b+|\omega| x}\right)^{l+1} \quad \text { and } \quad\left|\mathcal{R}_{1, x}(\omega, x)\right| \leq \frac{\widetilde{C}_{1}}{|\omega|^{2}}\left(\frac{x}{b+|\omega| x}\right)^{l}
$$


Indeed, consider

$$
r_{0}(x):=\int_{0}^{x} G_{l}(\omega, x, t) 2 A_{2}^{\prime}(t) \frac{\sqrt{t} J_{l+3 / 2}(\omega t)}{\omega^{l+3 / 2}} d t \quad \text { and } \quad r_{n+1}(x):=\int_{0}^{x} G_{l}(\omega, x, t) q(t) r_{n}(t) d t .
$$

Then using the inequalities (7.11), (7.12), (7.13) we obtain that

$$
\left|r_{0}(x)\right| \leq 2 A_{2} C C_{1} \sqrt{\frac{2}{\pi}}\left(\frac{x}{b+|\omega| x}\right)^{l+1} \int_{0}^{x}\left(\frac{t}{b+|\omega| t}\right)^{2} \theta(t) d t \leq \frac{C_{2}}{\omega^{2}}\left(\frac{x}{b+|\omega| x}\right)^{l+1},
$$

where we used that $\frac{t}{b+|\omega| t} \leq \frac{1}{|\omega|}$ and $\int_{0}^{x} \theta(t) d t \leq \int_{0}^{b} \theta(t) d t<\infty$. It follows by induction that

$$
\left|r_{n}(x)\right| \leq \frac{C_{2}}{n !|\omega|^{2}}\left(\frac{x}{b+|\omega| x}\right)^{l+1}\left(C \int_{0}^{x} \frac{t \tilde{q}(t)}{b+|\omega| t} d t\right)^{n},
$$

where $\tilde{q}(t)$ is the same as in the proof of Proposition 4.1. Summing up all the functions $r_{n}$ we obtain the first estimate in (17.14) with $\tilde{C}:=C_{2} \exp \left(C \int_{0}^{b} \frac{t \tilde{q}(t)}{b+|\omega| t} d t\right)<\infty$. The second estimate follows directly from the first estimate and (7.10).

Differentiating (4.18) with respect to $x$ we obtain that

$$
\mathcal{R}_{1, x}(\omega, x)=u_{l}^{\prime}(\omega, x)-d(\omega)\left(\omega b_{l}^{\prime}(\omega x)+\frac{Q(x)}{2} b_{l}(\omega x)\right)-\frac{A_{0} \sqrt{x} J_{l+3 / 2}(\omega x)}{4 \omega^{l+3 / 2}}\left(2 q(x)-\frac{(2 l+1) Q(x)}{x}\right),
$$

where we used the formula $J_{\nu}^{\prime}(z)=J_{\nu-1}(z)-\frac{\nu}{z} J_{\nu}(z)$. Consider the function

$$
g_{2}(\omega):=u_{l}^{\prime}(\omega, x)-d(\omega)\left(\omega b_{l}^{\prime}(\omega x)+\frac{Q(x)}{2} b_{l}(\omega x)\right) .
$$

As follows from (17.14), $\left|\mathcal{R}_{1, x}(\omega, x)\right| \leq \frac{\widetilde{C}_{1}}{\omega^{l+2}}$. The last term in $(\underline{7.15})$ decays as $\omega^{-l-2}$ when $|\omega| \rightarrow \infty$. Hence the function $g_{2}(\omega)$ also decays as $\omega^{-l-2}$. Moreover, $g_{2}(\omega)$ is an entire even function of the complex variable $\omega$ and similarly to the proof of Proposition 4.1 we obtain the existence and smoothness of the function $\widetilde{R}^{(2)}$.

From (7.5) similarly to the proof of Theorem 4.3 we obtain

$$
\gamma_{n}(x)=(4 n+1) \int_{0}^{x} R^{(2)}(x, t) P_{2 n}\left(\frac{t}{x}\right) d t=(4 n+1) \sum_{k=0}^{n} \frac{l_{2 k, 2 n}}{x^{2 k}} \int_{0}^{x} R^{(2)}(x, t) t^{2 k} d t .
$$

In order to calculate the last integral we expand all the terms of the equality (7.4) into the series with respect to $\omega$ (for the function $u_{l}^{\prime}$ we differentiate (3.11)) and compare coefficients at equal powers of $\omega$. We obtain that

$$
\int_{0}^{x} R^{(2)}(x, t) t^{2 k} d t=\varphi_{k}^{\prime}(x)-c_{k, l} x^{2 k+l}\left((2 k+l+1)+\frac{x Q(x)}{2}\right) .
$$

Substitution of this expression into (7.16) gives us (7.6).

Convergence of the series (17.5) and the estimates (17.7) and (7.8) can be obtained similarly to the proof of Theorem 4.3 .

Suppose that $q \in C^{2 p+1}[0, b]$. Consider the asymptotic expansion (4.18) with $m=p+1$. The remainder $\mathcal{R}_{p+1}$ satisfies equations similar to (7.9) and (7.10). Applying the successive approximations method one can obtain the following estimate for the derivative $\left|\mathcal{R}_{p+1, x}(\omega, x)\right| \leq$ 
$\frac{C_{3}}{|\omega|^{p+2}}\left(\frac{x}{b+|\omega| x}\right)^{l}$. Observe that $\left(A_{k}(x) \frac{\sqrt{x} J_{l+k+1 / 2}(\omega x)}{\omega^{l+k+1 / 2}}\right)^{\prime}=\left(A_{k}^{\prime}(x) \sqrt{x}-\frac{(l+k) A_{k}(x)}{\sqrt{x}}\right) \frac{J_{l+k+1 / 2}(\omega x)}{\omega^{l+k+1 / 2}}+$ $A_{k}(x) \sqrt{x} \frac{J_{l+k-1 / 2}(\omega x)}{\omega^{l+k-1 / 2}}$, i.e., for each fixed $x$ the expressions $g_{2}$ and $\mathcal{R}_{p+1, x}$ differ by a linear combination of the terms $\frac{J_{l+k+1 / 2}(\omega x)}{\omega^{l+k+1 / 2}}$. Now the last statement of the theorem can be obtained following the proof of Proposition 4.5.

Theorem 7.2. Under the conditions of Theorem 7.1, the $x$-derivative of the regular solution $u_{l}(\omega, x)$ of (3.1) satisfying the asymptotic relation $u_{l}(\omega, x) \sim x^{l+1}$ when $x \rightarrow 0$ has the form

$$
u_{l}^{\prime}(\omega, x)=d(\omega)\left(\omega b_{l}^{\prime}(\omega x)+\frac{Q(x)}{2} b_{l}(\omega x)\right)+\sum_{n=0}^{\infty}(-1)^{n} \gamma_{n}(x) j_{2 n}(\omega x)
$$

where the coefficients $\gamma_{n}$ are defined by (7.6). For the difference between $u_{l}^{\prime}(\omega, x)$ and

$$
\stackrel{\circ}{u}_{l, N}(\omega, x):=d(\omega)\left(\omega b_{l}^{\prime}(\omega x)+\frac{Q(x)}{2} b_{l}(\omega x)\right)+\sum_{n=0}^{N}(-1)^{n} \gamma_{n}(x) j_{2 n}(\omega x)
$$

the following inequalities are valid

$$
\left|u_{l}^{\prime}(\omega, x)-\stackrel{\circ}{u}_{l, N}(\omega, x)\right| \leq \sqrt{x} \varepsilon_{N}(x) \quad \text { for all } \omega \in \mathbb{R},
$$

and

$$
\left|u_{l}^{\prime}(\omega, x)-\stackrel{\circ}{u}_{l, N}(\omega, x)\right| \leq\left(\frac{\sinh (2 C x)}{2 C}\right)^{1 / 2} \varepsilon_{N}(x) \quad \text { for all } \omega \in \mathbb{C},|\operatorname{Im} \omega| \leq C, C \geq 0,
$$

where $\varepsilon_{N}$ is a sufficiently small nonnegative function such that $\left\|R^{(2)}(x, \cdot)-R_{N}^{(2)}(x, \cdot)\right\|_{L_{2}[0, x]} \leq$ $\varepsilon_{N}(x)$, which exists due to Theorem 7.1.

Proof. Substitution of (7.5) into (17.4) together with the formula 2.17.7 from [27, p. 433] gives us (7.17). The inequalities (7.19) and (7.20) are obtained in a complete analogy with the proof of Theorem 5.1 .

\section{Recurrent equations for $\gamma_{n}$}

From (5.1) we have that

$$
u_{l}^{\prime}(\omega, x)=\omega d(\omega) b_{l}^{\prime}(\omega x)+\sum_{n=0}^{\infty}(-1)^{n}\left(\beta_{n}^{\prime}(x) j_{2 n}(\omega x)-\omega \beta_{n}(x) j_{2 n+1}(\omega x)+\frac{2 n}{x} \beta_{n}(x) j_{2 n}(\omega x)\right) .
$$

Comparing this expression with (7.17) we obtain the equality

$$
\begin{aligned}
& \frac{d(\omega) Q(x)}{2} b_{l}(\omega x)+\sum_{n=0}^{\infty}(-1)^{n} \gamma_{n}(x) j_{2 n}(\omega x) \\
& \quad=\sum_{n=0}^{\infty}(-1)^{n}\left(\beta_{n}^{\prime}(x) j_{2 n}(\omega x)-\omega \beta_{n}(x) j_{2 n+1}(\omega x)+\frac{2 n}{x} \beta_{n}(x) j_{2 n}(\omega x)\right) .
\end{aligned}
$$


Using (6.1) and rearranging the terms we arrive at the equality

$$
\frac{d(\omega) Q(x) b_{l}(\omega x)}{2 \omega x}-\left(\beta_{0}^{\prime}(x)-\gamma_{0}(x)\right) j_{-1}(\omega x)=\sum_{n=1}^{\infty} \tilde{\alpha}_{n}(x) j_{2 n-1}(\omega x)
$$

where

$$
\tilde{\alpha}_{n}:=(-1)^{n}\left[\frac{1}{4 n+1}\left(\beta_{n}^{\prime}-\gamma_{n}+\frac{2 n}{x} \beta_{n}\right)-\frac{1}{4 n-3}\left(\beta_{n-1}^{\prime}-\gamma_{n-1}-\frac{2 n-1}{x} \beta_{n-1}\right)\right]
$$

for $n=1,2, \ldots$ and $\beta_{0}^{\prime}-\gamma_{0}=x^{l+1} Q / 2$.

Comparison of (8.1) with (6.2) and application of a similar procedure to that from Section 6 leads to the relations

$$
\begin{aligned}
\frac{\pi \tilde{\alpha}_{n}(x)}{(4 n-1)} & =Q(x) \int_{0}^{\infty} \frac{d(\omega) b_{l}(\omega x)}{\omega} j_{2 n-1}(\omega x) d \omega \\
& =\frac{Q(x)}{4} \frac{x^{l+1} \sqrt{\pi} \Gamma(l+2) \Gamma(l+3 / 2) \Gamma(n-1 / 2)}{\Gamma(l-n+2) \Gamma(n+1) \Gamma(n+l+3 / 2)}
\end{aligned}
$$

and hence

$$
\tilde{\alpha}_{n}(x)=\frac{(4 n-1)}{4 \sqrt{\pi}} \frac{Q(x) x^{l+1} \Gamma(l+2) \Gamma(l+3 / 2) \Gamma(n-1 / 2)}{\Gamma(l-n+2) \Gamma(n+1) \Gamma(n+l+3 / 2)}=: C_{n} Q(x) x^{l+1} .
$$

Equations (8.2) together with the recursive formulas (6.9)-(6.11) can be used to calculate the coefficients $\gamma_{n}$ alternatively to the formulas (7.6). We start with

$$
\gamma_{0}=\beta_{0}^{\prime}+x^{l+1} Q / 2=u_{0}^{\prime}-(l+1) x^{l}+x^{l+1} Q / 2
$$

and compute recursively for $n \geq 1$

$$
\begin{aligned}
\gamma_{n}= & \frac{4 n+1}{4 n-3}\left[\gamma_{n-1}+(4 n-1)\left(\frac{2 u_{0}^{\prime} \theta_{n}}{x^{2 n}}+\frac{2 \eta_{n}}{u_{0} x^{2 n}}-\frac{\beta_{n-1}}{x}\right)\right] \\
& +(-1)^{n}(4 n+1)\left[\frac{B_{n}}{x^{2 n}}\left(\mu_{n} u_{0}^{\prime}+\frac{\kappa_{n}}{u_{0}}\right)-C_{n} Q(x) x^{l+1}\right] .
\end{aligned}
$$

\section{$9 \quad$ Numerical results}

The main ingredients for the construction and application of the approximate solution $u_{l ; N}$ and its approximate derivative $\stackrel{\circ}{u}_{l, N}$ are the coefficients $\beta_{n}$ and $\gamma_{n}$. Unfortunately, we are not aware of any single non-zero potential $q$ for which one can obtain these coefficients in a closed form. They have to be calculated numerically. Our experiments show that even hundreds of the coefficients $\beta_{n}$ and $\gamma_{n}$ can be easily computed within seconds without any difficulty. Below we explain some details. We also refer the reader to [6], 22] and [18] where many aspects of the numerical implementation are discussed in detail.

The first coefficients $\beta_{0}$ and $\gamma_{0}$ are given by (6.7) and (8.3) in terms of the particular solution $u_{0}$ of equation (3.5) satisfying asymptotic conditions (3.3) and (3.4). Such solution together with its derivative can be computed using the SPPS representation [6, Section 3]. The assumption for

the solution $u_{0}$ to be non-vanishing automatically holds if $q(x) \geq 0, x \in(0, b]$. For other cases one may need to apply the spectral shift technique as described in [6], [7]. 
As was mentioned in [18] for the non-singular case, the direct formulas lead to a rapid growth of the error in the computed coefficients $\beta_{k}$ and $\gamma_{k}$ and are not recommended for numerics. The same happens with the formulas (4.9) and (7.6), they allow one to calculate only 10-15 coefficients $\beta_{k}$ and $\gamma_{k}$ in the machine precision. However one still may apply them when arbitrary precision arithmetics is used. In the present paper we neither utilize nor present any illustration of the numerical performance of the formulas (4.9) and (7.6).

Instead, the recurrent formulas (6.9) -(6.11) and (8.4) show an excellent computational stability allowing one to compute easily even hundreds of the coefficients. All the functions involved were represented by their values on the uniform mesh. We used a somewhat overwhelming number of mesh points (like 20-50 thousands) in order to make the integration errors negligible and to concentrate mainly on the numerical performance of the proposed formulas. It is worth emphasizing that even in this case all the reported calculations took only several seconds. The integrals in (6.9) and (6.10) were calculated using the modified 6 point Newton-Cottes rule. This rule consists in interpolating the function values at these 6 points by a fifth order polynomial and using the integral of this polynomial as the approximation for the indefinite integral.

We would like to point out that the numerical integration in (6.10) may be tricky due to the division by $u_{0}^{2}$, a function behaving near zero as $x^{2 l+2}$. Even small errors in the values of the functions $\eta_{n}$ and $\kappa_{n}$ near zero can lead to large erroneous values after dividing by $u_{0}^{2}$. As a workaround we chose the following strategy. We simply ignored (replaced by 0) several first values of the integrands in (6.10) prior to numerical integration. Due to 6 point integration rule utilized, we used the following simple cut-off criterion. For every 6 integrand values $y_{0}, \ldots, y_{5}$ on the consecutive mesh points we calculated the expression

$$
\Delta_{5}:=y_{0}-5 y_{1}+10 y_{2}-10 y_{3}+5 y_{4}-y_{5}
$$

(related to the fifth order divided difference) and compared it to the two smallest absolute values of the numbers $y_{0}, \ldots, y_{5}$. We started the integration rule from the first 6 -tuple for which the quantity $\left|\Delta_{5}\right|$ was not significantly larger than the two smallest absolute values. This simple criterion resulted to be sufficient to deliver acceptable numerical results.

\subsection{Analysis of the decay rate of the coefficients $\beta_{n}$ and $\gamma_{n}$}

Absolute values of the coefficients $\beta_{k}$ decrease as $k \rightarrow \infty$, see the estimates (4.12), (4.15) and (4.17). However due to the presence of the term $\frac{4 n+1}{4 n-3} \beta_{n-1}$ in the formula (6.11) the error in one computed coefficient $\beta_{n}$ propagates to all further coefficients. I.e., when one computes large numbers of the coefficients $\beta_{n}$, their absolute values reach some floor value and stabilize. The same happens with the coefficients $\gamma_{n}$. A simple error measure can be derived taking $t=x$ in (4.8) and (7.5). One has

$$
\sum_{n=0}^{\infty} \frac{\beta_{n}(x)}{x}=R(x, x)=0 \quad \text { and } \quad \sum_{n=0}^{\infty} \frac{\gamma_{n}(x)}{x}=R^{(2)}(x, x)=0,
$$

and the discrepancy of the truncated series from zero provides some insight on how good the approximation is.

Consider $b=\pi$ and $q=x^{2}$ in (1.1). We computed the coefficients $\beta_{n}$ for $n \leq 100$ for several different values of the parameter $l$. On Figure 1 we present the plot of the values $\left|\beta_{n}(\pi)\right|$ vs. $n$. We chose log-log scale graph to reveal a possible power law decay rate of the coefficients. As one can see from the graph, the absolute values $\left|\beta_{n}(\pi)\right|$ indeed obey a power law decay whenever $l \notin \mathbb{N}$, and a faster than polynomial decay for $l \in \mathbb{N}$, c.f., Proposition 4.5. We estimated the decay rate degree $r$ in the power law $\left|\beta_{n}(\pi)\right| \approx c n^{-r}$ and obtained that $r \approx 2 l+3$ for $l \notin \mathbb{N}$, c.f., (4.17). 


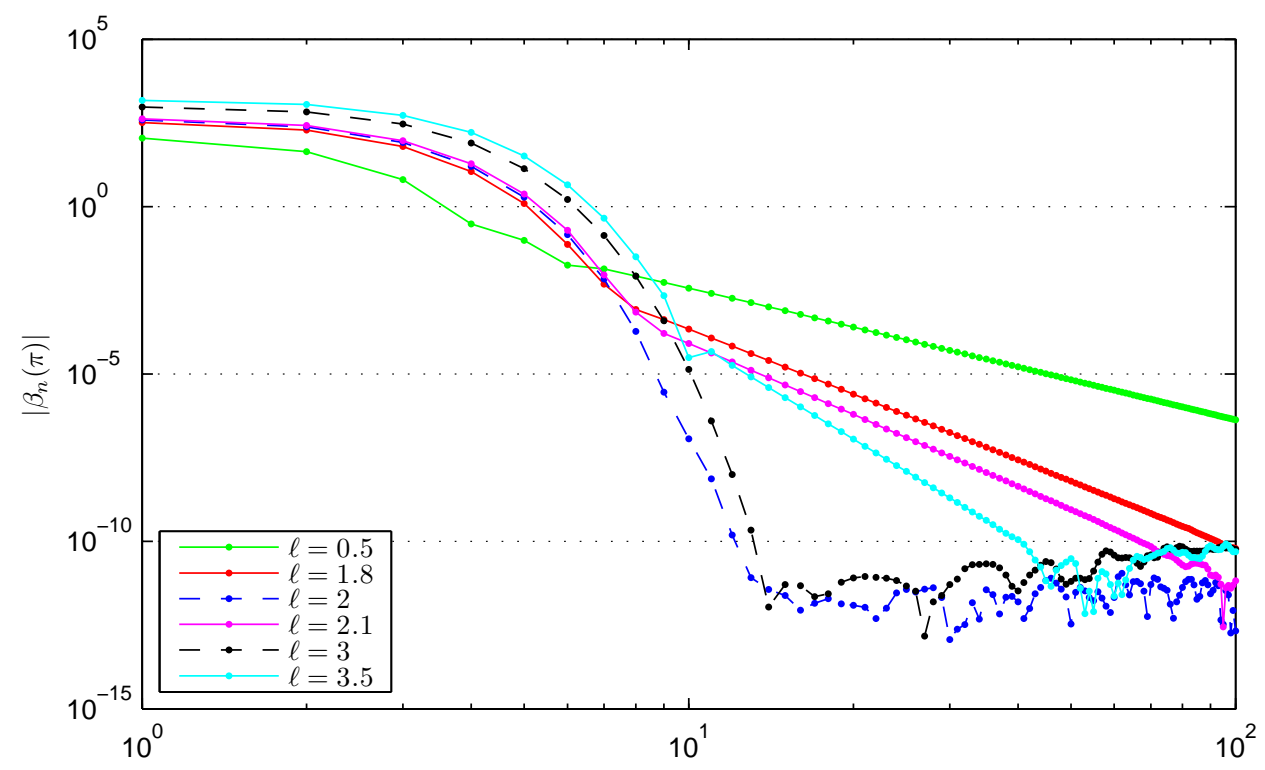

Figure 1: Illustration of the decay of the numbers $\left|\beta_{n}(\pi)\right|$ for $q(x)=x^{2}$ and for different values of the parameter $l$ in (1.1). Dashed lines correspond to integer values of $l$, solid lines correspond to non-integer values of $l$.

However we observed that the smoothness requirements on the potential $q$ in Proposition 4.5 and Theorem 7.1 look to be excessive. For that we considered several potentials,

$$
q_{1}(x)=x^{2}, \quad q_{2}(x)=\sqrt{\pi^{2}-x^{2}}, \quad q_{3}(x)=\frac{1}{x}, \quad q_{k}(x)= \begin{cases}0, & x \leq \pi / 2 \\ (x-\pi / 2)^{k-4}, & x>\pi / 2 \\ & k \in\{4,5,6\}\end{cases}
$$

computed the coefficients $\beta_{n}$ and $\gamma_{n}, n \leq 100$ for different non integer values of $l$ and found the degrees $r$ and $s$ in the power law approximations $\left|\beta_{n}(\pi)\right| \approx c_{1} n^{r}$ and $\left|\gamma_{n}(\pi)\right| \approx c_{2} n^{s}$. The obtained values of $r$ and $s$ are presented on Figure 2 ,

The first potential illustrates that one can not expect an improvement of the estimate (4.17) even for infinitely smooth potentials (c.f., Remark 4.6). However, it is illustrated by the potentials $q_{2}$ and $q_{3}$ that the coefficients $\beta_{n}$ and $\gamma_{n}$ can decay as (or closely to) $n^{-2 l-3}$ even for potentials possessing singularities or unbounded derivatives at the endpoints. The situation changes when the potentials are not sufficiently smooth inside the interval $(0, b)$, as illustrated by $q_{4}, q_{5}$ and $q_{6}$. For small values of the parameter $l$ the coefficients $\beta_{n}$ and $\gamma_{n}$ still decay as $n^{-2 l-3}$, while for larger values of $l$ the decay rate degree becomes smaller.

For integer values of $l$ the estimate (4.15) predicts a faster than polynomial decay of the coefficients $\beta_{n}$ in the case of a $C^{\infty}$-potential and guaranties a polynomial decay rate for potentials of finite smoothness. We verified this numerically considering the potentials

$$
q_{k}(x)= \begin{cases}1, & x \leq \pi / 2 \\ 1+(x-\pi / 2)^{k}, & x>\pi / 2, \quad k=0, \ldots, 5\end{cases}
$$

and comparing the degree of decay rate as in the previous experiments. On Figure 3 we present the plots of the values $\left|\beta_{n}(\pi)\right|$ and $\left|\gamma_{n}(\pi)\right|$ vs. $n$. For all calculations we took $l=2$. As one can see from the plots, the slope of the lines (corresponding to the decay rate degree) indeed increases 

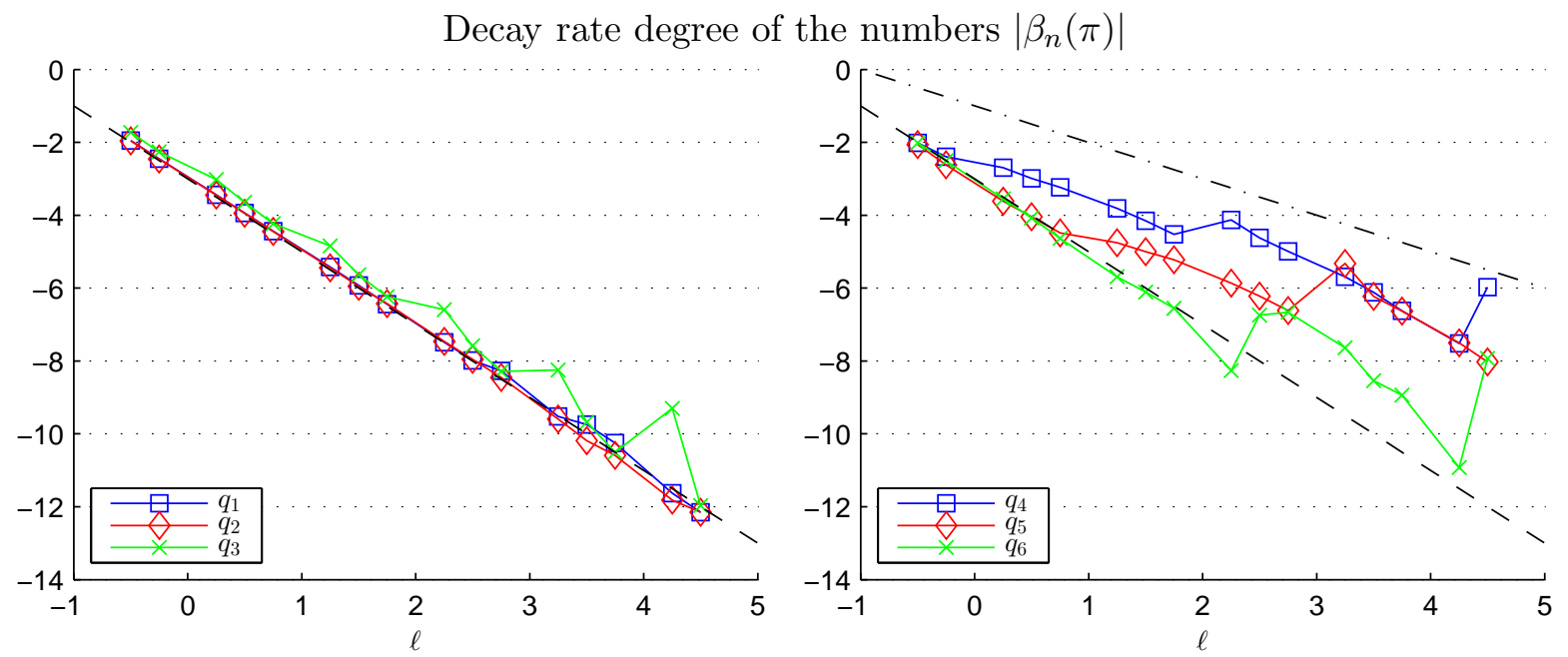

Decay rate degrees of the numbers $\left|\gamma_{n}(\pi)\right|$
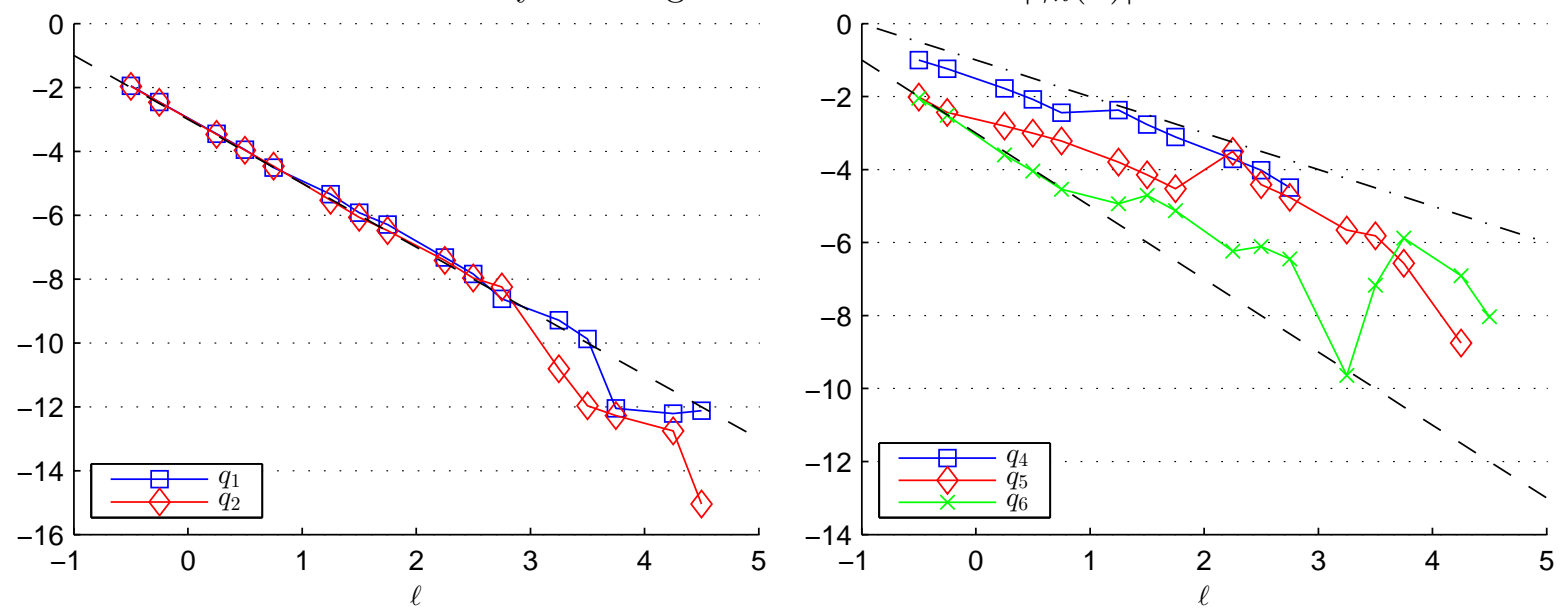

Figure 2: Illustration of the decay rate degree of the numbers $\left|\beta_{n}(\pi)\right|$ (two upper plots) and $\left|\gamma_{n}(\pi)\right|$ (two lower plots) for different potentials $q_{i}, i=1, \ldots, 6$ given by (9.2) as functions of the parameter $l$. Additionally we plot the lines $y=-2 l-3$ (dashed line) and $y=-l-1$ (dash-dot line).

when the potential smoothness increases by 2, as predicted by Proposition 4.5, however as in the previous experiment, the increase of the slope is by 2 , not by 1 .

A more detailed study of the observed phenomena is left for a future work.

\subsection{Solution of spectral problems}

One of the possible applications of the proposed representations (5.1) and (7.17) is to the approximate solution of spectral problems. We emphasize that the idea of this subsection is to illustrate the convergence estimates and error bounds from Theorem 5.1 and Theorem 7.1 rather than to compete with the best available software packages such as MATSLise [24]. Our implementation of the approximate method based on the proposed formulas is straightforward. Clearly the method can benefit, e.g., from interval subdivision techniques combined with the representation proposed in [18], and we are sure that a robust software package can be created, however we left these tasks for future research.

In all performed numerical experiments the coefficients $\beta_{n}$ and $\gamma_{n}$ were computed as was ex- 

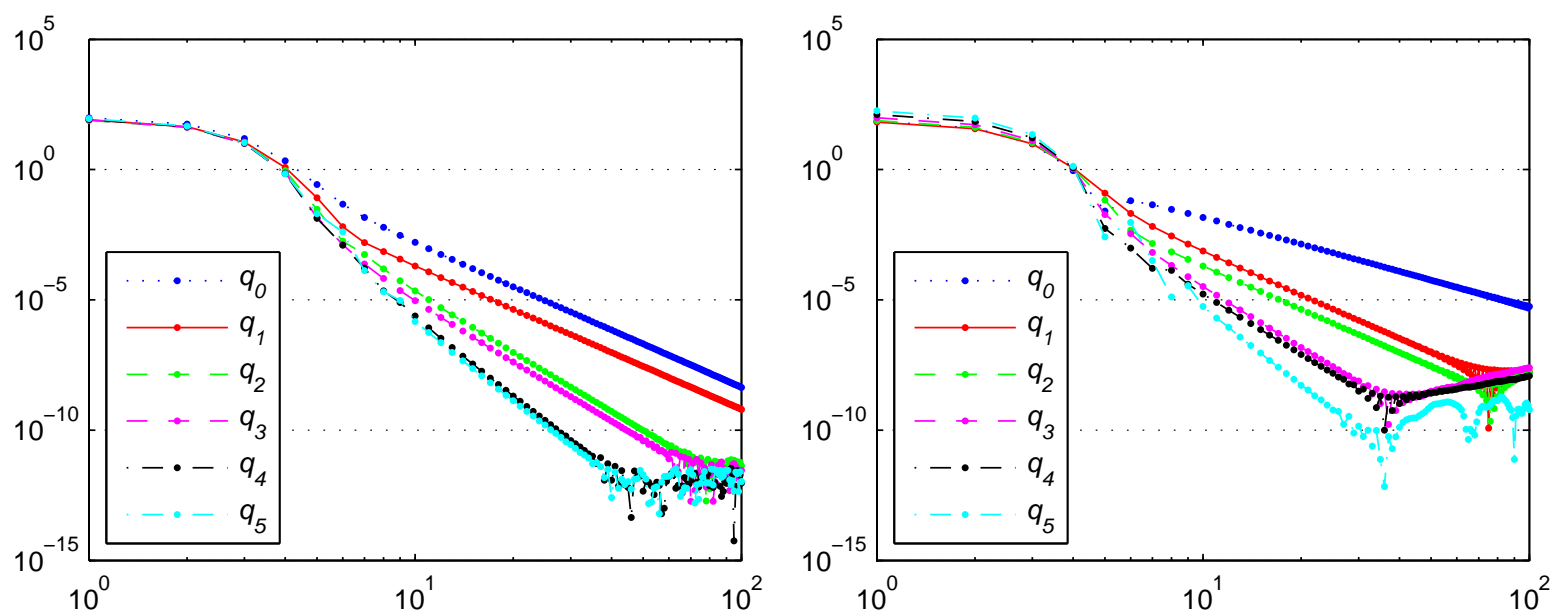

Figure 3: Illustration of the decay rate of the numbers $\left|\beta_{n}(\pi)\right|$ (left plot) and $\left|\gamma_{n}(\pi)\right|$ (right plot) for different potentials $q_{k}, k=0, \ldots, 5$ given by (9.3).

plained at the beginning of this section. Matlab 2012 in machine precision was used. The optimal number $N$ of terms for the approximations (5.2) and (17.18) was estimated using the formulas (9.1) for $x=b$, as the value when the partial sums of the series in (9.1) reach the machine-precision induced floor. The upper values like $N=169$ in the following examples appear due to our straightforward implementation of the formula (6.11), larger values of $N$ cause the machine precision overflow in computation of $\Gamma(N+l+3 / 2)$. In all the proposed spectral problems Wolfram Mathematica 8 was able to find the regular solution in the explicit form, which was used to calculate the exact eigenvalues.

Example 9.1. Consider the following spectral problem

$$
\begin{gathered}
-u^{\prime \prime}+\left(\frac{l(l+1)}{x^{2}}+x^{2}\right) u=\omega^{2} u, \quad 0 \leq x \leq \pi, \\
u(\omega, \pi)=0 .
\end{gathered}
$$

The value $l=3 / 2$ was considered in [5, Example 2] and [6, Example 7.3]. We compared the results with those obtained using (5.2) with $N=100$. Exact eigenvalues together with the absolute errors of the approximate eigenvalues obtained using different methods are presented in Table 1. The proposed method is abbreviated as NSBF (from Neumann series of Bessel functions). As one can see from the results, the proposed method is comparable with the SPPS method for lower-index eigenvalues and is clearly superior for the 100th eigenvalue. Additionally it is much faster than the SPPS method.

On Figure 4 we present the absolute errors of the computed eigenvalues for different values of $l$. One can see that the obtained errors follow theoretical predictions given in Proposition 4.5, Theorem 5.1 and Remark 5.2. That is, for integer values of $l$, only few coefficients $\beta_{k}$ were used (small values of $N$ on the left plot) due to the rapid decrease of them, while for non-integer values of $l$ larger numbers of the coefficients were necessary (large values of $N$ on the right plot). The better accuracy of the first 90-100 eigenvalues on the right plot is due to the estimate (5.5). The fast deterioration of the accuracy for higher eigenvalues for $l=5$ and $l=10$ is explained in Remark 5.2 .

Example 9.2. Consider the same equation as in Example 9.1 with a different boundary condition:

$$
u^{\prime}(\omega, \pi)=0 .
$$




\begin{tabular}{cccccc}
\hline$n$ & $\omega_{n}$ (Exact/MATSLISE) & $\Delta \omega_{n}(\mathrm{NSBF})$ & $\Delta \omega_{n}(\mathrm{SPPS})$ & $\Delta \omega_{n}($ SLEIGN2) & $\Delta \omega_{n}([\underline{5}])$ \\
\hline 1 & 2.46294997397397 & $1.4 \cdot 10^{-14}$ & $2.7 \cdot 10^{-13}$ & $5.4 \cdot 10^{-8}$ & $9.4 \cdot 10^{-7}$ \\
2 & 3.28835292994256 & $5.2 \cdot 10^{-14}$ & $6.7 \cdot 10^{-12}$ & $1.8 \cdot 10^{-7}$ & $1.4 \cdot 10^{-5}$ \\
3 & 4.14986421874478 & $1.2 \cdot 10^{-13}$ & $8.2 \cdot 10^{-13}$ & $5.0 \cdot 10^{-7}$ & $3.1 \cdot 10^{-5}$ \\
5 & 6.00758145811600 & $6.6 \cdot 10^{-13}$ & $5.0 \cdot 10^{-13}$ & $2.2 \cdot 10^{-6}$ & $4.1 \cdot 10^{-6}$ \\
7 & 7.93973737689930 & $2.9 \cdot 10^{-13}$ & $6.0 \cdot 10^{-13}$ & $7.3 \cdot 10^{-6}$ & \\
10 & 10.8861250916173 & $1.5 \cdot 10^{-12}$ & $8.6 \cdot 10^{-13}$ & $2.4 \cdot 10^{-5}$ & \\
20 & 20.8202301908124 & $1.4 \cdot 10^{-11}$ & $9.6 \cdot 10^{-14}$ & $3.4 \cdot 10^{-4}$ & \\
30 & 30.7973502195868 & $1.5 \cdot 10^{-11}$ & $1.9 \cdot 10^{-12}$ & $1.6 \cdot 10^{-3}$ & \\
50 & 50.7786768095149 & $8.7 \cdot 10^{-11}$ & $1.3 \cdot 10^{-10}$ & $1.0 \cdot 10^{-2}$ & \\
100 & 100.764442245651 & $9.4 \cdot 10^{-9}$ & $5.3 \cdot 10^{-4}$ & & \\
\hline
\end{tabular}

Table 1: The eigenvalues from Example 9.1 for $l=3 / 2$ compared to the results reported in [6]. Since eigenvalues produced by the MATSLISE package coincide with the exact eigenvalues to all reported digits, we present them in the combined column. $\Delta \omega_{n}$ denotes the absolute error of the computed eigenvalue $\omega_{n}$.
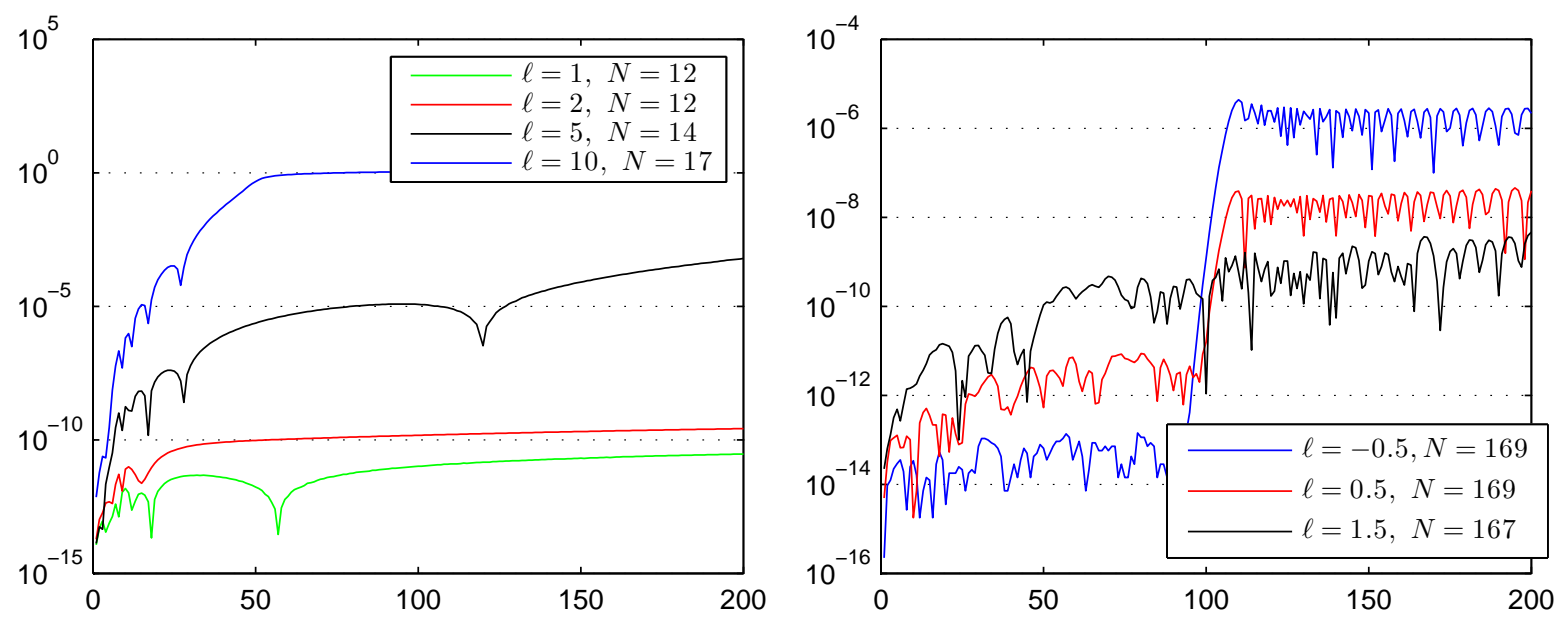

Figure 4: Absolute errors of the first 200 eigenvalues for the spectral problem from Example 9.1 for different values of $l$. On the legends the number $N$ used for calculation of the approximate solution (5.2) is shown next to the value of the parameter $l$.

Absolute errors of the obtained eigenvalues are presented on Figure 5, Again, the results follow the theoretical predictions from Theorem 7.1 .

Example 9.3. Consider the spectral problem for the hydrogen atom equation [5, Example 4], [6, Example 7.4]

$$
\begin{aligned}
-u^{\prime \prime}+\left(\frac{l(l+1)}{x^{2}}+\frac{1}{x}\right) u & =\omega^{2} u, \quad 0 \leq x \leq \pi, \\
u(\omega, \pi) & =0 .
\end{aligned}
$$

Absolute errors of the obtained eigenvalues are presented on Figure 6. The singularity in the potential presents no difficulty for the proposed method. However the case $l=1$, contrary to the previous examples, requires more coefficients $\beta_{n}$ for the approximate solution (5.2) to be computed. They do not decay equally fast for integer values of $l$. 


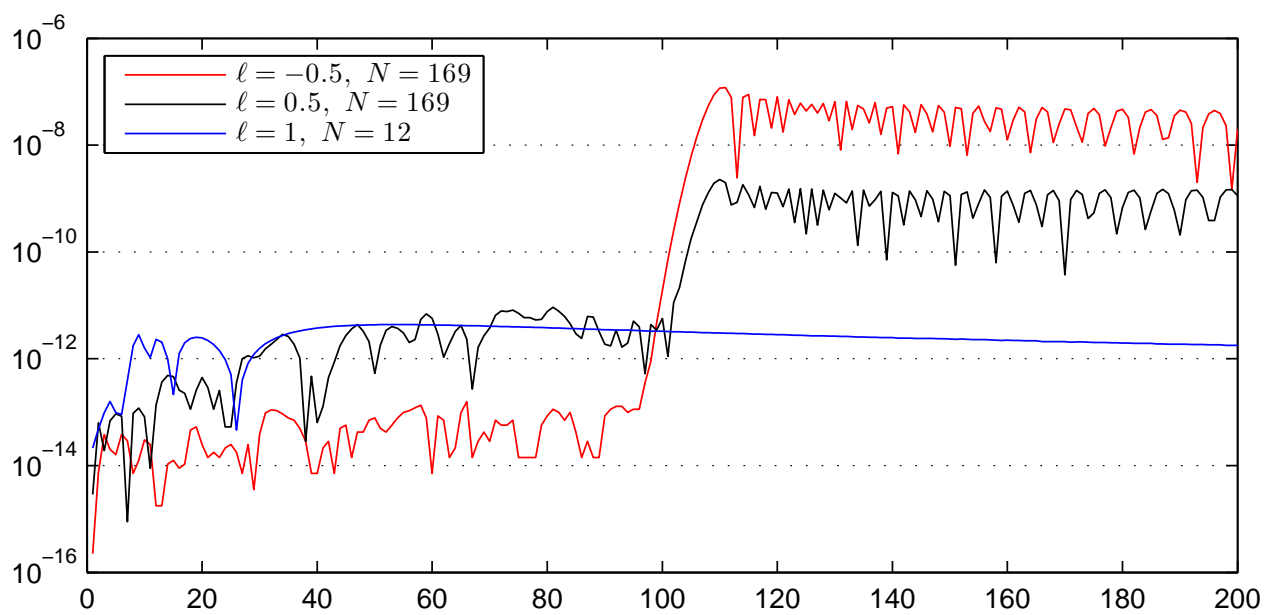

Figure 5: Absolute errors of the first 200 eigenvalues for the spectral problem from Example 9.2 for different values of $l$. On the legend the number $N$ used for calculation of the approximate solution (5.2) is shown next to the value of the parameter $l$.

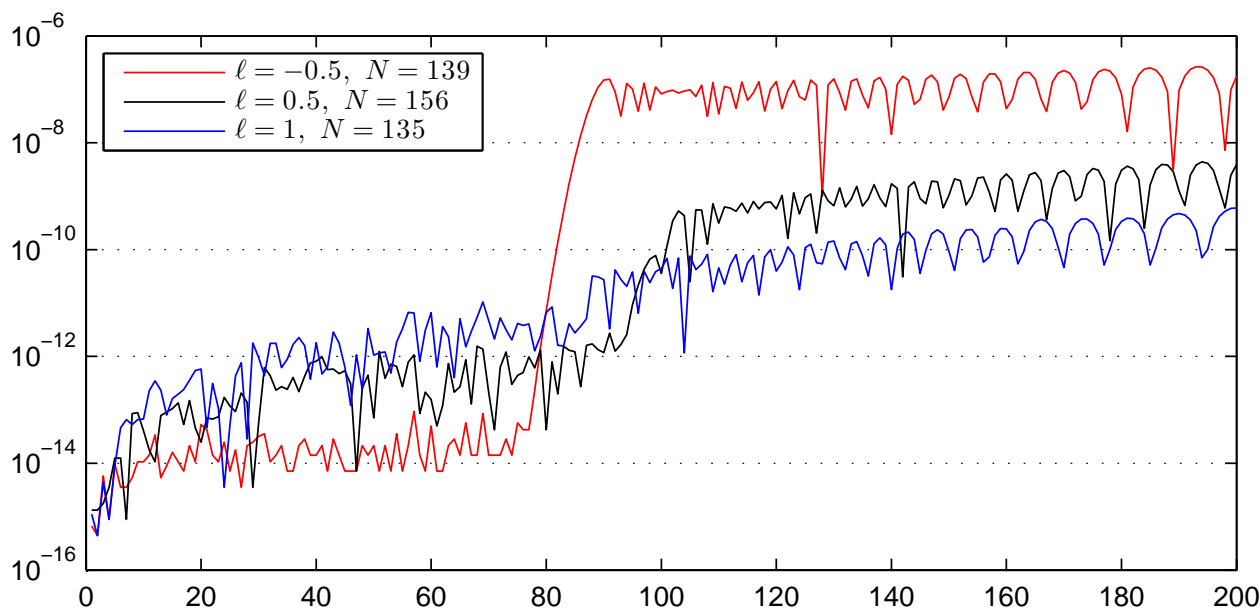

Figure 6: Absolute errors of the first 200 eigenvalues for the spectral problem from Example 9.3 for different values of $l$. On the legend the number $N$ used for calculation of the approximate solution (5.2) is shown next to the value of the parameter $l$.

\section{References}

[1] M. Abramovitz and I. A. Stegun, Handbook of mathematical functions, New York: Dover, 1972.

[2] R. A. Adams, Sobolev Spaces. Pure and Applied Mathematics, Vol. 65, New York-London: Academic Press, 1975.

[3] A. Baricz, D. Jankov and T. K. Pogány, Neumann series of Bessel functions, Integral Transforms Spec. Funct. 23 (2012), no. 7, 529-538.

[4] H. Blancarte, H. Campos and K. Khmelnytskaya, Spectral parameter power series method for discontinuous coefficients, Math. Methods Appl. Sci. 38 (2015), no. 10, 2000-2011.

[5] A. Boumenir and B. Chanane, Computing eigenvalues of Sturm-Liouville systems of Bessel type, $\mathrm{P}$. Edinburgh Math. Soc. 42 (1999), 257-265.

[6] R. Castillo-Pérez, V. V. Kravchenko and S. M. Torba, Spectral parameter power series for perturbed Bessel equations, Appl. Math. Comput. 220 (2013), 676-694. 
[7] R. Castillo-Pérez, V. V. Kravchenko and S. M. Torba, Analysis of graded-index optical fibers by the spectral parameter power series method, J. Optics 17 (2015), 025607 (9pp).

[8] H. Chébli, A. Fitouhi and M. M. Hamza, Expansion in series of Bessel functions and transmutations for perturbed Bessel operators, J. Math. Anal. Appl. 181 (1994), no. 3, 789-802.

[9] M. Coz and Ch. Coudray, The Riemann solution and the inverse quantum mechanical problem, J. Math. Phys. 17 (1976), no. 6, 888-893.

[10] R. A. DeVore and G. G. Lorentz, Constructive Approximation, Berlin: Springer-Verlag, 1993, x+449p.

[11] A. Fitouhi and M. M. Hamza, A uniform expansion for the eigenfunction of a singular second-order differential operator, SIAM J. Math. Anal. 21 (1990), 1619-1632.

[12] J.-C. Guillot and J. V. Ralston, Inverse spectral theory for a singular Sturm-Liouville operator on [0,1], J. Differential Equations 76 (1988), no. 2, 353-373.

[13] D. Jackson, The theory of approximation. Reprint of the 1930 original, American Mathematical Society, Providence, RI, 1994.

[14] Y. Katznelson, An introduction to harmonic analysis. Third edition, Cambridge: Cambridge University Press, 2004. xviii+314pp.

[15] A. Kostenko, A. Sakhnovich and G. Teschl, Inverse eigenvalue problems for perturbed spherical Schrödinger operators, Inverse Problems 26 (2010), 105013 (14pp).

[16] A. Kostenko and G. Teschl, On the singular Weyl-Titchmarsh function of perturbed spherical Schrödinger operators, J. Differential Equations 250 (2011), 3701-3739.

[17] K. V. Khmelnytskaya, V. V. Kravchenko and H. C. Rosu, Eigenvalue problems, spectral parameter power series, and modern applications, Math. Methods Appl. Sci. 38 (2015), 1945-1969.

[18] V. V. Kravchenko, L. J. Navarro and S. M. Torba, Representation of solutions to the one-dimensional Schrödinger equation in terms of Neumann series of Bessel functions, submitted, available at arXiv:1508.02738

[19] V. V. Kravchenko and R. M. Porter, Spectral parameter power series for Sturm-Liouville problems, Math. Methods Appl. Sci. 33 (2010), 459-468.

[20] V. V. Kravchenko, S. M. Torba and J. Yu. Santana-Bejarano, Generalized wave polynomials and transmutations related to perturbed Bessel equations, submitted, available at arXiv:1606.07850,

[21] V. V. Kravchenko and S. M. Torba, Transmutations and spectral parameter power series in eigenvalue problems, Oper. Theory Adv. Appl. 228 (2013), 209-238.

[22] V. V. Kravchenko and S. M. Torba, Analytic approximation of transmutation operators and applications to highly accurate solution of spectral problems, J. Comput. Appl. Math. 275 (2015) 1-26.

[23] N. M. Lebedev, Special Functions and Their Applications, New York: Dover, 1972.

[24] V. Ledoux and M. Van Daele, Matslise 2.0: A Matlab toolbox for Sturm-Liouville computations, ACM Trans. Math. Softw. 42 (2016), 29:1-18.

[25] K. Okamoto, Fundamentals of optical waveguides, San Diego: Academic Press, 2000.

[26] F. Olver, Asymptotics and Special Functions, Wellesley, Massachusets: A K Peters, 1997.

[27] A. P. Prudnikov, Yu. A. Brychkov and O. I. Marichev, Integrals and series. Vol. 2. Special functions, New York: Gordon \& Breach Science Publishers, 1986, 750 pp.

[28] J. Yu. Santana-Bejarano, Operadores de transmutación para la ecuación de Bessel perturbada y aproximación analítica de sus soluciones, PhD Thesis, CINVESTAV del IPN, 2016.

[29] S. M. Sitnik, On solution to the problem of unitary generalization to the Sonine-Poisson transmutations, Belgorod State University Scientific Bulletin, Mathematics and Physics, 5(76) (2010), No 18, 135-153 (in Russian).

[30] P. K. Suetin, Classical orthogonal polynomials, 3rd ed. (in Russian), Moscow: Fizmatlit, 2005, 480 pp.

[31] E. C. Titchmarsh, Introduction to the theory of Fourier integrals. Third edition, New York: Chelsea Publishing Co., 1986. x+394 pp.

[32] H. Triebel, Interpolation theory, function spaces, differential operators. Second edition, Amsterdam-New York: North-Holland Publishing Co., 1978, 528 pp. 
[33] V. Ya. Volk, On inversion formulas for a differential equation with a singularity at $x=0$, Uspehi Matem. Nauk (N.S.) 8 (1953). no. 4(56), 141-151.

[34] G. N. Watson, A Treatise on the theory of Bessel functions, 2nd ed., reprinted, Cambridge: Cambridge University Press, 1996, vi+804 pp.

[35] J. Weidmann, Spectral Theory of Ordinary Differential Operators, Lecture Notes in Math., Berlin: Springer, vol. 1258, 1987.

[36] J. E. Wilkins, Neumann series of Bessel functions, Trans. Amer. Math. Soc. 64 (1948), 359-385. 